Draft VERSion July 28, 2021

Preprint typeset using LATEX style emulateapj v. 7/15/03

\title{
SUPERNOVA SIMULATIONS WITH BOLTZMANN NEUTRINO TRANSPORT: A COMPARISON OF METHODS
}

\author{
M. Liebendörfer ${ }^{1,2,3}$, M. RAmpP ${ }^{4}$, H.-Th. JAnkA ${ }^{4}$, A. MEzzACAPPA ${ }^{2}$ \\ Draft version July 28, 2021
}

\begin{abstract}
Accurate neutrino transport has been built into spherically symmetric simulations of stellar core collapse and postbounce evolution. The results of such simulations agree that spherically symmetric models with standard microphysical input fail to explode by the delayed, neutrino-driven mechanism. Independent groups implemented fundamentally different numerical methods to tackle the Boltzmann neutrino transport equation. Here we present a direct and detailed comparison of such neutrino radiation-hydrodynamical simulations for two codes, AGILE-BOLTZTRAN of the Oak RidgeBasel group and VERTEX of the Garching group. The former solves the Boltzmann equation directly by an implicit, general relativistic discrete angle method on the adaptive grid of a conservative implicit hydrodynamics code with second-order TVD advection. In contrast, the latter couples a variable Eddington factor technique with an explicit, moving-grid, conservative high-order Riemann solver with important relativistic effects treated by an effective gravitational potential. The presented study is meant to test both neutrino radiation-hydrodynamics implementations and to provide a data basis for comparisons and verifications of supernova codes to be developed in the future. Results are discussed for simulations of the core collapse and post-bounce evolution of a $13 \mathrm{M}_{\odot}$ star with Newtonian gravity and a $15 \mathrm{M}_{\odot}$ star with relativistic gravity.

Subject headings: supernovae: general-neutrinos — radiative transfer-hydrodynamics — relativitymethods: numerical
\end{abstract}

\section{INTRODUCTION}

Computer simulations are becoming more and more popular. They allow investigations of physics on office desks rather than explorations through hands-on experiments (This does not imply a transition from hard work to gaming). In the industrial context, the two approaches are not separable: the computer codes have to be validated. After a computer design has been completed, its relation to reality will inevitably be assessed in the manufacture and evaluation of prototypes. How about the growing importance of computer simulations in astrophysics - where are the measurements found to test aspects of a complex computer code in idealized setups, and where are the prototypes that validate the quality of the results in the targeted application? The first step of code development is accompanied by the verification of partial aspects of the code in simplified test problems where the solution is analytically known. The code can be improved step by step. Additionally, laboratory experiments may be used to further verify the code with accurate measurements in idealized setups. The transition to code validation is made when its capability to handle complicated coupled processes is tested and the completeness of the physical description is evaluated. In the industrial context, this is achieved by more comprehensive experiments and measurements, or, ultimately, by the comparison of computer designs with the prop-

1 CITA, University of Toronto, Toronto, Ontario M5S 3H8, Canada

2 Physics Division, Oak Ridge National Laboratory, Oak Ridge, Tennessee 37831-6354

3 Department of Physics and Astronomy, University of Tennessee, Knoxville, Tennessee 37996-1200

4 Max-Planck-Institut für Astrophysik, Karl-SchwarzschildStrasse 1, D-85741 Garching, Germany erties of manufactured prototypes. We would now be tempted to relate the validation of computer generated results with real life prototypes in manufacturing to the comparison of astrophysical simulations with astronomical observations. This would, however, circumvent the goal of astrophysical simulations: One does not assume unknown physics in industrial design. Perfect agreement between a computer simulation and the behavior of a prototype can indeed be seen as proof of the quality of the computer code. The situation is different in astrophysics, where the understanding of the physics of an event is rather the goal than the ingredient. The comparison between simulation and observation is essential to demonstrate the physical understanding of the event, it cannot at the same time be used to qualify code performance. The gap in code validation between detached analytical test calculations and the astrophysical application can be bridged by code comparisons (Calder et al. 2002), based on the assumption that different numerical approaches are likely to show different strengths and weaknesses in simulations of complex physical systems. Differences in the simulation results are an indicator for uncertainties in the numerical methods.

In the present paper we document the detailed comparison of results from different supernova codes. Both of our codes aim to provide a solution to the Boltzmann neutrino transport equation in spherical symmetry. This is achieved by fundamentally different numerical methods: The code of the Oak Ridge-Basel group (AGILE-BOLTZTRAN) consists of a general relativistic time-implicit discrete-angle $\left(\mathrm{S}_{N}\right)$ Boltzmann solver, which is coupled in an operator split fashion to a general relativistic time-implicit hydrodynamics solver with a dynamical adaptive grid. It implements a direct finite difference representation of the Boltzmann equation 
(Mezzacappa \& Bruenn 1993a; Mezzacappa \& Messer 1999; Liebendörfer. Rosswog. \& Thielemann 2002; Liebendörfer et al. 2004). The Garching code, VERTEx, is a one-dimensional version of a program that was developed to perform multi-dimensional supernova simulations with accurate ray-by-ray neutrino transport. It is based on the explicit, moving-grid, finite-volume hydrodynamics code PROMETHEUs, which employs a Riemann solver for constructing the solution of the hydrodynamics equations. The neutrino transport is handled in an operator-split step and is calculated by a variable Eddington factor closure of neutrino energy, number, and momentum equations, where the variable Eddington factor is derived from the formal solution of a spherically averaged model Boltzmann equation (Rampp \& Janka 2002). In spherical symmetry, there is only one "ray" for the solution of the moments equations and no spherical averaging is necessary for the model Boltzmann equation. Therefore, a convergence in the iterations between the moments equations and the closure from the model Boltzmann equation provides in spherical symmetry a solution of the complete Boltzmann equation.

This work has two main goals. (i) The direct comparison of two codes applied to the same challenging astrophysical scenario with concerted physics (spherical symmetry, progenitor models, nuclear and weak interaction physics, general relativistic effects). (ii) The production of reference results to test future supernova codes in the spherical limit. Machine-readable data files are included in the electronic edition of the Journal.

Neutrinos play a crucial role in collapsing cores of massive stars. The loss of electron lepton number by the production and escape of electron neutrinos determines the collapse dynamics and the position where the supernova shock forms. Energy and lepton number transport by neutrino diffusion also govern the evolution of the nascent neutron star. The energy transfer by neutrinos to the medium that surrounds the protoneutron star may revive the stalled accretion front and thus drive a delayed explosion (Wilson 1985; Bethe \& Wilson 1985). Neutrino interactions moreover set the protonto-nucleon ratio and therefore the conditions for nucleosynthesis in the innermost supernova ejecta. Sustained energy deposition near the protoneutron star surface causes a post-explosion outflow of baryonic matter, the so-called neutrino-driven wind, which is discussed as a potential site for the formation of r-process elements (Wooslev et al. 1994; Takahashi. Witti. \& Janka 1994; Sumivoshi et al. 2000; Wanaio et al. 2001; Thompson. Burrows, \& Mever 2001).

Deep inside the protoneutron star the absorption and scattering mean free paths of neutrinos are very small and therefore neutrinos diffuse and are in chemical equilibrium with the stellar plasma. With decreasing density the neutrino interaction lengths become larger, before, finally the neutrinos can stream freely. Since the reaction cross sections rise steeply with the neutrino energy, lowenergy neutrinos decouple from the stellar background at higher densities. Most electron flavor neutrinos emerge from the accreting material at the base of the cooling region under semi-transparent conditions and propagate to the heating region where their angular distribution influences the energy deposition behind the accretion front.
Neither diffusion nor free streaming is a good approximation in this important region where neutrinos strongly couple the dynamics of different layers on a short propagation time scale.

An accurate treatment of the neutrino transport and of neutrino-matter interactions therefore requires the combination of neutrino sources at one location with neutrino opacities at other locations as described by the energy- and angle-dependent Boltzmann transport equation. The solution of the Boltzmann equation is also desirable to test approximations, the most elaborate of which are certainly multigroup flux-limited diffusion (Bruenn 1985; Mvra et al 1987; Bruenn. DeNisco, \& Mezzacappa 2001) and twomoment closure schemes (Bludman \& Cernohorskv 1995; Smit. Cernohorskv. \& Dullemond 1997). With the growing computer capability it has become feasible to provide solutions to the Boltzmann transport equation not only for the collapse phase (Mezzacappa \& Bruenn 1993a), but also in consistent dynamical simulations of the post-bounce evolution (Rampp \& Janka 2000; Mezzacappa et al. 2001; Liebendörfer et al. 2001; Thompson. Burrows. \& Pinto 2003).

The paper is organized as follows. We will describe in Sect. 2 the stellar models and the physical ingredients that constitute the problem to be solved by our numerical methods. In Sect. 3 we will briefly resume characteristic features and capabilities of both neutrino radiationhydrodynamics codes. In Sect. 4 our results for the two considered stellar models will be discussed with special focus on the differences between the runs. In Sect. 5 we shall summarize our findings and draw conclusions.

\section{DESCRIPTION OF THE MODEL}

\subsection{Progenitors}

We present in this paper two different models. One of them includes only the most essential physical ingredients; e.g., the transport of electron flavor neutrinos and antineutrinos, but not the heavy-lepton neutrinos and antineutrinos. It is meant to serve as a guideline for future code development and transport approximations. The model is based on a $13 \mathrm{M}_{\odot}$ progenitor of Nomoto \& Hashimoto (1988). The $13 \mathrm{M}_{\odot}$ progenitor model has a tradition in supernova simulations, its exceptionally small iron core sustained the hope to produce prompt explosions. We call this run N13, as it is based on Newtonian gravity. A second run was launched from a $15 \mathrm{M}_{\odot}$ progenitor of Wooslev \& Weaver (1995). This progenitor has been widely used in supernova investigations, as it provides a model of a massive star in the middle of the mass range that is expected to end its life in a supernova. The run takes into account all neutrino flavors with "standard" input physics as listed in Bruenn (1985). General relativistic effects are included in this physically more complete run, G15, and the input physics has been extended by ion-ion correlations and nucleon-nucleon bremsstrahlung.

\subsection{Radiation Hydrodynamics in Spherical Symmetry}

The stellar progenitor model is evolved in time by means of the hydrodynamics and neutrino transport equations. Since there is no danger of grid entangling in spherical symmetry, we make use of the free- 
dom in AGILE-BOLTZTRAN to choose orthogonal comoving space-time coordinates and describe the interactions in the collision term in the most convenient comoving frame for the neutrino four-momentum (see e.g., Cardall \& Mezzacappa (2003) for a generalized discussion of coordinate choices for the radiation transport). In the metric of Misner \& Sharp (1964); Mav \& White (1966),

$d s^{2}=-\alpha^{2}(c d t)^{2}+\left(\frac{1}{\Gamma} \frac{\partial r}{\partial a}\right)^{2} d a^{2}+r^{2}\left(d \vartheta^{2}+\sin ^{2} \vartheta d \varphi^{2}\right)$,

the equations of hydrodynamics in spherical symmetry in the presence of a radiation field can be written in the form (Lindquist 1966; Liebendörfer. Mezzacappa, \& Thielemann 2001)

$$
\begin{aligned}
& \frac{\partial}{\partial t}\left[\frac{\Gamma}{\rho}\right]=\frac{\partial}{\partial a}\left[4 \pi r^{2} \alpha u\right] \\
& \frac{\partial}{\partial t}\left[\Gamma\left(1+\frac{e+J}{c^{2}}\right)+\frac{u H}{c^{4}}\right]= \\
& -\frac{\partial}{c^{2} \partial a}\left[4 \pi r^{2} \alpha(u p+u \rho K+\Gamma \rho H)\right] \\
& \frac{\partial}{\partial t}\left[u\left(1+\frac{e+J}{c^{2}}\right)+\frac{\Gamma H}{c^{2}}\right]= \\
& -\frac{\partial}{\partial a}\left[4 \pi r^{2} \alpha\left(\Gamma p+\Gamma \rho K+\frac{u \rho H}{c^{2}}\right)\right] \\
& -\frac{\alpha}{r}\left[\left(1+\frac{e+3 p / \rho+J+3 K}{c^{2}}\right) \frac{G m}{r}\right. \\
& -\left(1-\frac{2 G m}{r c^{2}}\right)(J-3 K)-2\left(\frac{p}{\rho}+K\right) \\
& \left.+8 \pi r^{2} \frac{G}{c^{2}}\left(\left(1+\frac{e+J}{c^{2}}\right)(p+\rho K)-\frac{\rho H^{2}}{c^{4}}\right)\right] \\
& \frac{\partial}{\partial a}\left[\frac{4 \pi}{3} r^{3}\right]=\frac{\Gamma}{\rho} \\
& \frac{\partial m}{\partial a}=\Gamma\left(1+\frac{e+J}{c^{2}}\right)+\frac{u H}{c^{4}} \\
& \frac{\partial}{\partial t}\left[\frac{1}{4 \pi r^{2} \rho} \frac{H}{c^{4}}\right]= \\
& -\left(1+\frac{e+J}{c^{2}}\right) \frac{\partial \alpha}{\partial a}-\frac{1}{\rho c^{2}} \frac{\partial}{\partial a}[\alpha(p+\rho K)] \\
& +\frac{\alpha \Gamma}{4 \pi r^{3} \rho c^{2}}(J-3 K) \text {. }
\end{aligned}
$$

The metric is based on an enclosed baryon mass label, $a$, and a coordinate time, $t$. It refers to the areal radius, $r$, the "Lorentz" factor, $\Gamma=\sqrt{1+(u / c)^{2}-2 G m /\left(r c^{2}\right)}$, and the lapse function, $\alpha$. The angles $\vartheta$ and $\varphi$ describe a two-sphere. The quantity $u=\alpha^{-1} \partial r / \partial t$ takes the place of a fluid velocity and $m$ is the enclosed gravitational mass, proportional to the enclosed total energy. Both of our codes split these equations into hydrodynamics equations and transport equations. VERTEX employs Eulerian coordinates which can be obtained by a coordinate transformation. The fluid is specified in its rest frame by the rest mass density, $\rho$, specific internal energy, $e$, and electron number fraction, $Y_{e}$, for which an additional evolution equation (lepton number conservation) is solved.
The hydrodynamics equations are closed by the equation of state, which gives the pressure, $p$, as a function of density, internal energy, and electron number. The zeroth, $J$, first, $H / c$, and second, $K$, angular moment of the monochromatic neutrino intensity (normalized by the rest-mass density) are determined by the transport equation which includes the interactions listed in Table 1 in the collision integral. Eq. (7) determines the lapse function. Eq. (6) allows us to integrate outward from $a=0$ to obtain the total energy; it translates to the Poisson equation in the Newtonian limit. Eq. (5) defines the general relativistic analogue to the Newtonian volume. Eq. (41) describes the change of radial momentum; to leading order it is proportional to $-G m /(c r)^{2}+(J-3 K) / r$. Eq. (3) describes the evolution of the total energy. Note that there are no contributions from terms of zeroth and first order in $c$. Eq. (2) relates the evolution of the specific volume to the divergence of the velocity field.

\subsection{Nuclear and Weak Interaction Physics Input}

The equation of state describes the thermodynamic state of a fluid element based on density, $\rho$, temperature, $T$, and the composition. The relation between the specific internal energy and the pressure closes the system of hydrodynamics equations. For this comparison we use the equation of state of Lattimer \& Swesty (1991). It assumes nuclear statistical equilibrium and is based on a liquid drop model for a representative nucleus with atomic number $A$ and charge $Z$, surrounded by free alpha particles, protons, and neutrons. These baryons are immersed in an electron and positron gas that equilibrates with a photon gas by the pair creation process. The incompressibility modulus can be adjusted. We use a value of $K=180 \mathrm{MeV}$. Above nuclear density, where no isolated individual nuclei are present, the transition to a proton-neutron-electron gas is made by a Maxwell phase transition. In any of these cases, the nuclear composition at given temperature and density is determined by the specification of the electron fraction $Y_{e}$. At low densities/temperatures the VERTEX code switches to an equation of state that considers electrons, positrons, photons, nucleons and nuclei consistent with the composition used in the progenitor model (Rampp \& Janka 2002). The switch is triggered in N13 and G15 by a density threshold of $\rho<6 \times 10^{7} \mathrm{~g} / \mathrm{cm}^{3}$. AGILE-BOLTZTRAN applies the same switch in the run N13, but considers in the low density domain only one nucleus, ${ }^{28} \mathrm{Si}$. In run G15, silicon is converted to NSE under energy conservation at a burning temperature of $0.44 \mathrm{MeV}$ (Mezzacappa et al. 2001).

As for the neutrino-matter interactions, we have chosen to use the conventional ("standard") opacities, i.e., a description which follows closely the one detailed by Bruenn (1985); Mezzacappa \& Bruenn (1993c). Note, however, that there are small differences in the neutrino description employed by the two groups. While AGILEBOLTZTRAN treats the $\mu / \tau$ neutrinos and antineutrinos separately, they are combined to one species in VERTEX. In order to save computer time, usually only electron neutrinos are considered in the VERTEX calculations during the core collapse phase. Tests have shown that taking into account also electron antineutrinos and the heavylepton neutrinos leads to only minuscule differences in this phase of the supernova evolution (their inclusion in 
TABLE 1

OVERVIEW OF ALL NEUTRINO-MATTER INTERACTIONS CONSIDERED IN THE N13 RUNS.

\begin{tabular}{|c|c|}
\hline Reaction $^{a}$ & Reference \\
\hline$\nu A \rightleftharpoons \nu A$ & Bruenn (1985) (no ion-ion correlations!) \\
\hline$\nu \mathrm{N} \rightleftharpoons \nu \mathrm{N}$ & Bruenn (1985); Mezzacappa \& Bruenn (1993c) \\
\hline$\nu_{\mathrm{e}} \mathrm{n} \rightleftharpoons \mathrm{e}^{-} \mathrm{p}$ & Bruenn (1985); Mezzacappa \& Bruenn (1993c) \\
\hline $\bar{\nu}_{\mathrm{e}} \mathrm{p} \rightleftharpoons \mathrm{e}^{+} \mathrm{n}$ & Bruenn (1985); Mezzacappa \& Bruenn (1993c) \\
\hline$\nu_{\mathrm{e}} A^{\prime} \rightleftharpoons \mathrm{e}^{-} A$ & Bruenn (1985); Mezzacappa \& Bruenn (1993c) \\
\hline$\nu \bar{\nu} \rightleftharpoons \mathrm{e}^{-} \mathrm{e}^{+}$ & Bruenn (1985) \\
\hline$\nu \mathrm{e}^{ \pm} \rightleftharpoons \nu \mathrm{e}^{ \pm}$ & $\begin{array}{l}\text { Mezzacappa \& Bruenn (1993c); } \\
\text { Cernohorsky (1994) }\end{array}$ \\
\hline
\end{tabular}

${ }^{a}$ In the first column the symbol $\nu$ represents any of the neutrinos $\nu_{\mathrm{e}}, \bar{\nu}_{\mathrm{e}}$ (heavy-lepton neutrinos are neglected in N13). The symbols $\mathrm{e}^{-}, \mathrm{e}^{+}, \mathrm{n}, \mathrm{p}$ and $A$ denote electrons, positrons, free neutrons and protons, and heavy nuclei, respectively, the symbol $\mathrm{N}$ means $\mathrm{n}$ or $\mathrm{p}$. The references point to papers where information can be found about the approximations employed in the rate calculations. Details about the numerical implementation can be found in the methodical papers of Rampp \& Janka (2002) and Mezzacappa \& Messer (1999); Liebendörfer et al. (2004), respectively. The G15 runs additionally include reactions with $\mu$ - and $\tau$-neutrinos and their antiparticles, ion-ion correlation effects in neutrino-nuclei interactions, and nucleon-nucleon bremsstrahlung.

the postbounce phase, however, is important as demonstrated by the comparison between models N13 and G15 in Sect. 4). The Garching group routinely includes nucleon-nucleon bremsstrahlung as a source (or sink) for neutrino-antineutrino pairs. The decrease of coherent neutrino scattering off heavy ions due to ion-ion correlations and electron screening (Itoh 1975; Horowitz 1997; Bruenn \& Mezzacappa 1997) is also taken into account. These improvements have been switched off in the N13 models and added to AGILE-BOLTZTRAN such that they are consistently included in both G15 runs. In both codes, the implementation of the ion-ion correlations has been updated with the structure function given in Itoh et al. (2004). Because of the large mass contrast between species with $A \leq 4$ and the representative heavy nucleus we omit the somewhat arbitrary averaging of the effect over species and consider only the representative nucleus for the calculation of the ion separation.

\section{NUMERICAL METHODS}

The two codes follow very different approaches to evaluate the radiation moments $J, H$, and $K$. Contrary to flux-limiting and "gray" transport methods, neither of our methods needs to make assumptions about the angular or the spectral distribution of the radiation field. Important features and implementation details of the two codes are summarized in the following subsections.

\subsection{AGILE-BOLTZTRAN}

The concept and first implementation of BOLTZTRAN has been developed in Mezzacappa \& Bruenn $(1993 \mathrm{a} \mathrm{b}$ c) for core collapse simulations in the order $v / c$ approximation. Essential for the computational efficiency of the implicit scheme is the storage of the interactions in a dynamical table which delivers consistent derivatives of all cross sections and thermodynamical quantities for the Newton-Raphson iterations in the solution of the nonlinear equations (Mezzacappa \& Messer 1999; Messer 2000). For the highly dynamical situation after bounce, BOLTZTRAN has been coupled to the hydrodynamics code AGILE. The finite differencing has been revised for energy conservation and extended to solve the general relativistic Eqs. (2 8) (Liebendörfer 2000; Liebendörfer. Rosswog. \& Thielemann 2002; Liebendörfer et al. 2004).

\subsubsection{Hydrodynamics}

The hydrodynamics part of the Lagrangian Eqs. (2.7) is solved by implicit conservative finite differencing. One strength is the implementation of a dynamically moving adaptive grid following Winkler, Norman, \& Mihalas (1984) and Dorfi \& Drurv (1987). In the general relativistic extension, it is equivalent to a resolutiondependent choice of shift vectors, that allow a continuous coordinate translation in the radial direction during the evolution of the model. Artificial viscosity has been included in a consistent, but causality violating manner based on the tensor viscosity of Tscharnuter \& Winkler (1979). It provides the mechanism for energy dissipation in the shock front and defines the shock width such that the number of attracted adaptive grid points does not grow beyond limits. A major advantage of the adaptive grid is the dynamical allocation of computational zones to regions where they are needed. The zoning for hydrodynamics and neutrino transport is always kept congruent for consistency reasons. During the evolution of the model, one group of grid points follows the accretion front, while another resolves the steep density gradient between the outer layers of the protoneutron star and the infalling matter, where most of the electron flavor neutrinos stream away. The use of artificial viscosity is not a disadvantage of the method, as it only plays a minor local role in stabilizing the shock front (the shock width is set to a few percent of the shock radius and captured by $\sim 10$ moving grid points). A disadvantage of the adaptive grid approach in an earlier implementation (see Liebendörfer. Rosswog. \& Thielemann (2002) for a detailed description) is the numerical diffusion introduced by the first-order advection in regions where the adaptive grid does not concentrate its zones, e.g. in a rarefaction wave or at sharp discontinuities of the composition. During this comparison, improvement has been achieved by upgrading AGILE with second order total variation diminishing (TVD) advection based on a Van Leer flux limiter.

\subsubsection{Neutrino Transport}

The neutrino transport part, BOLTZTRAN, solves the Boltzmann transport equation,

$$
\begin{aligned}
C & =\frac{\partial F}{\alpha c \partial t}+\frac{\mu}{\alpha} \frac{\partial}{\partial a}\left[4 \pi r^{2} \alpha \rho F\right] \\
& +\Gamma\left(\frac{1}{r}-\frac{1}{\alpha} \frac{\partial \alpha}{\partial r}\right) \frac{\partial}{\partial \mu}\left[\left(1-\mu^{2}\right) F\right] \\
& +\left(\frac{\partial \ln \rho}{\alpha c \partial t}+\frac{3 u}{r c}\right) \frac{\partial}{\partial \mu}\left[\mu\left(1-\mu^{2}\right) F\right] \\
& -\mu \Gamma \frac{1}{\alpha} \frac{\partial \alpha}{\partial r} \frac{1}{E^{2}} \frac{\partial}{\partial E}\left[E^{3} F\right] \\
& +\left(\mu^{2}\left(\frac{\partial \ln \rho}{\alpha c \partial t}+\frac{3 u}{r c}\right)-\frac{u}{r c}\right) \frac{1}{E^{2}} \frac{\partial}{\partial E}\left[E^{3} F\right],
\end{aligned}
$$

in a finite difference representation implementing the discrete ordinates, or $\mathrm{S}_{N}$, method. The evolved quantity 
is the neutrino distribution function, $F(t, a, \mu, E)$, as a function of time $t$, rest mass $a$ enclosed in a sphere at radius $r$, propagation angle cosine $\mu$ with respect to radial direction, and neutrino energy $E$. Neutrinos in specific beams are created and destroyed according to the collision term, $C$, which includes the interactions listed in Table1 It is assumed that the neutrinos propagate freely between interactions. The free-particle motion along geodesics between collisions introduces the many correction terms apparent on the right hand side of Eq. (8). They stem from the use of spherical coordinates in combination with a description of the neutrino phase space in a comoving frame. Nevertheless, all terms can be labeled with a physical effect. In order of appearance in Eq. (8) these are, the time derivative of the neutrino distribution function, the propagation of neutrinos, the angle correction due to neutrino propagation, the angular aberration correction due to observer motion, the frequency shift in the gravitational potential, and the Doppler frequency shift due to observer motion. It is essential for the successful finite difference representation of Eq. (8) that it is upward compatible with simple special cases of the transport equation. Basic physical properties can be determined by the evaluation of expectation values with the neutrino distribution functions for various operators. The finite difference representation should support, e.g., the diffusion limit, total energy conservation, and the conservation of lepton number.

\subsubsection{Parameter settings}

Both runs, N13 and G15, were performed with 103 adaptive spatial zones ranging from the center of the progenitor star to about $7000 \mathrm{~km}$. A constant pressure boundary condition was used at the barely moving surface. The neutrino energy was resolved with 20 geometrically increasing energy groups, the first centered at 3 $\mathrm{MeV}$ and the last at $300 \mathrm{MeV}$. The propagation angle has been discretized with 6 angles suitable for Gaussian quadrature. Roughly 3000 time steps have been used for collapse and 7000 for the postbounce phase. The run N13 has been evolved with an order $v / c$ approximation of Eqs. (2 8) and run G15 with the general relativistic equations.

\subsection{VERTEX}

Independently from the efforts of the Oak RidgeBasel collaboration the Garching supernova group has treated the Boltzmann transport problem for neutrinos in core-collapse supernovae with a new variable Eddington factor method (Rampp 2000; Rampp \& Janka 2000, 2002), and has coupled it to the PROMETHEUS hydrodynamics code. The combined program allows for spherically symmetric (Rampp \& Janka 2000, 2002) as well as multi-dimensional simulations (Janka et al. 2004; Buras et al. 2003). The spherically symmetric "core" of the program, which was used for the calculations described below, will be referred to by the name VERTEX (Variable Eddington factor Radiative Transfer for supernova EXplosions).

\subsubsection{Hydrodynamics}

The integration of the equations of hydrodynamics is performed with the Newtonian finite-volume code
Prometheus (Frvxell. Müller, \& Arnett 1989) which was supplemented by additional problem specific features (Keil 1997). PROMETHEUs is a direct Eulerian, time-explicit implementation of the Piecewise Parabolic Method (PPM) of Colella \& Woodward (1984). As a Godunov scheme of third order in space and second-order in time with a Riemann solver, it is particularly well suited for following discontinuities in the fluid flow like shocks or boundaries between layers of different chemical composition. A notable advantage is its capability of tackling multi-dimensional problems with high computational efficiency and numerical accuracy. The code makes use of the "Consistent Multifluid Advection (CMA)" method (Plewa \& Müller 1999) for ensuring accurate advection of different chemical components in the fluid, and switches from the original PPM method to the more diffusive HLLE solver of Einfeldt (1988) in the vicinity of strong shocks to avoid spurious oscillations (the so-called "odd-even decoupling" phenomenon) when such shocks are aligned with one of the coordinate lines in multidimensional simulations Quirk 1994; Kifonidis 2000; Plewa \& Müller 2001).

\subsubsection{Neutrino transport}

The variable Eddington factor scheme for the neutrino transport, its coupling to the hydrodynamics part, and application to a number of test problems is described in much detail elsewhere (Rampp \& Janka 2002). Here we will only briefly summarize the characteristic features of the method. The coupled set of equations of hydrodynamics (Eqs. (1)-(4) in Rampp \& Janka (2002)) and radiation transport (Eqs. (6)-(8) ibidem) is equivalent to Eqs. (2)-(7) in the order $(v / c)$ limit. The equations are also split into a "hydrodynamics part" and a "neutrino part" and solved independently in subsequent ("fractional") steps. But the hydrodynamics and the transport solver can use radial grids and/or time steps that are different from each other.

In the neutrino transport method the integrodifferential character of the Boltzmann equation is tamed by applying a variable Eddington factor closure to the neutrino energy and momentum equations (and the simultaneously integrated first and second order moments equations for neutrino number). For this purpose the variable Eddington factor is determined from the formal solution of the Boltzmann equation on so-called "tangent rays". They coincide with radiation characteristics in Newtonian geometry. The system of the Boltzmann equation and its moments equations is iterated until convergence is achieved. The integration of the transport equations is implicit in time.

General relativistic effects are treated only approximately in the code (Rampp \& Janka 2002). The current version contains a modification of the gravitational potential by including correction terms due to pressure and energy of the stellar medium and neutrinos, which are deduced from a comparison of the Newtonian and relativistic equations of motion. The neutrino transport contains gravitational redshift and time dilation, but ignores the distinction between coordinate radius and proper radius. This simplification is necessary for coupling the transport code to the basically Newtonian hydrodynamics. We shall demonstrate in this paper that these approximations work satisfactorily well for the core collapse 
and the early postbounce phase (see Sect. 4.3). Moderate quantitative but no qualitative differences from the full relativistic treatment are mainly found at late times after the accretion front has started to retreat.

\subsubsection{Parameter settings}

For the neutrino transport in the N13 run an Eulerian radial grid with 235 radial zones (255 tangent rays) spaced logarithmically between 0 and $10000 \mathrm{~km}$ was used. The neutrino spectrum between 0 and $380 \mathrm{MeV}$ was discretized with 21 geometrically zoned energy bins, the center of the first bin being located at $2 \mathrm{MeV}$. The hydrodynamics, on the other hand, was solved on a radial grid of 400 zones which are moved with the stellar fluid during core collapse. Shortly after core bounce both radial grids were rezoned such that inside of a radius of 400 $\mathrm{km}$ the zones of the transport grid coincide with those of the hydro grid. For the post-bounce evolution the coordinates of the hydrodynamics grid (as well as those of the transport grid) remained fixed in time. Concerning the resolution and definition of numerical grids the same parameters were chosen in the G15 run with the exception that 19 energy bins between 0 and $380 \mathrm{MeV}$ were used instead of 21 groups and a rezoning of the radial grid was necessary at $\sim 200 \mathrm{~ms}$ after bounce. The new grid (hydrodynamics and neutrino transport) employs 40 additional radial zones in order to adequately represent the steepening density gradients at the surface of the nascent neutron star.

\section{COMPARISON OF THE RESULTS}

The simulations produce a sizeable amount of data, even if they are confined to one spatial dimension. Hence, we start with an overview of what we are going to compare and how the comparable quantities are derived from the code-specific results. During collapse and bounce it is quite natural to choose the enclosed baryon mass as spatial coordinate. It labels individual fluid elements and allows one to trace the history of each fluid element. Some tenths of milliseconds after bounce, the neutron star becomes rather static with mass accretion being essentially stationary. Therefore the presentation of the quantities as functions of radius is more appropriate. We present the three independent quantities that determine the thermodynamic state of the fluid element as measured by an observer comoving with the fluid: the rest mass density, the entropy per baryon, and the electron fraction. Furthermore, the radial velocity of the fluid element is displayed.

Note that in case of the relativistic model, G15, the coordinate independent change in areal radius per proper time, $u=\alpha^{-1} \partial r / \partial t$, is plotted in the (b)-panels of Figs. 8 , 9 11] and 12 for the AGILE-BOLTZTRAn run, whereas the velocity, $v=\partial r / \partial t$, is unchanged from the Newtonian case in the general relativistic approximation of VERTEX. Typical deviations of the metric coefficients from unity are shown in Table 2 In summary, these deviations are of order $1 \%$ in the preshock region, increase from $1 \%$ to $6 \%$ at the neutrinosphere during the simulation, and reach maximum values around $20 \%$ at the center of the star in case of the lapse function and around $10 \%$ close to the center in the case of $\Gamma$.

The neutrino transport quantities are represented by the neutrino luminosity and rms energy profiles as mea-
TABLE 2

Metric COEFFicients

\begin{tabular}{ccccccc}
\hline$t_{p b}[\mathrm{~ms}]$ & $\alpha_{\max }$ & $\alpha_{\nu}$ & $\alpha_{s}$ & $\Gamma_{\max }$ & $\Gamma_{\nu}$ & $\Gamma_{s}{ }^{a}$ \\
\hline-1 & 4 & 1 & - & 2 & 1 & - \\
0 & 15 & 1 & 3 & 8 & 2 & 5 \\
1 & 15 & 1 & 1 & 7 & 1 & 2 \\
5 & 15 & 1 & 1 & 7 & 1 & 1 \\
10 & 15 & 2 & 1 & 7 & 2 & 1 \\
50 & 17 & 3 & 0 & 7 & 3 & 1 \\
100 & 18 & 3 & 0 & 8 & 3 & 0 \\
250 & 23 & 6 & 0 & 10 & 6 & 1 \\
\hline
\end{tabular}

${ }^{a}$ Listed are the lapse function, $\alpha$, and the metric coefficient, $\Gamma$, by their deviations from unity in percent at several post-bounce times $t_{p b}$ during the G15 simulation with agile-Boltztran. The indices of $\alpha$ and $\Gamma$ refer to chosen locations where the values are given. The index $s$ corresponds to the position outside of the shock or accretion front, the index $\nu$ corresponds to the neutrinosphere of the heavy lepton neutrinos, and the index max stands for the maximum value in the whole star.

sured in the fluid rest frame. We also discuss selected quantities as functions of time, e.g. the trajectories of fluid elements, the position of the (accretion-)shock, the conditions at the center, or the neutrino luminosities and rms energies sampled at $500 \mathrm{~km}$ radius (as an approximation to radial infinity). The physical time in both runs is synchronized at bounce $(t=0)$, the moment when the central density reaches a local maximum immediately before the shock is formed. Negative times in the simulations therefore point to instances before bounce. We define the shock position, $R_{s}$, by the maximum of the velocity divergence (i.e. maximum compression, cf. Eq. (2)),

$$
x\left(R_{s}\right)=\max _{r}[x(r)], \quad x=-\frac{\partial\left(4 \pi r^{2} u\right)}{\partial V} .
$$

The luminosities and rms energies are given in the comoving frame as a function of the neutrino phase-space distribution function $F(t, a, \mu, E)$ according to

$$
\begin{aligned}
L(t, a) & =4 \pi r^{2} \rho \frac{2 \pi c}{(h c)^{3}} \int F(t, a, \mu, E) E^{3} d E \mu d \mu \\
\langle\epsilon(t, a)\rangle_{r m s} & =\sqrt{\frac{\int F(t, a, \mu, E) E^{4} d E d \mu}{\int F(t, a, \mu, E) E^{2} d E d \mu}} .
\end{aligned}
$$

In the general relativistic case, the definition of $F(t, a, \mu, E)$ in Eq. (8) implies that the luminosity at radius $r$ must be interpreted as originating from a neutrino number per proper time crossing a mass shell as measured by an observer comoving with the shell. The neutrinos carry energies, $E$, which are also measured in the comoving frame.

We compare time slices in our runs at three crucial instances in the postbounce evolution: Bounce $\left(t_{p b}=0\right.$ $\mathrm{ms})$, burst $\left(t_{p b}=3 \mathrm{~ms}\right)$, and broil $\left(t_{p b}>100 \mathrm{~ms}\right)$. The importance of core bounce, the instance when the infall is reversed at the center due to the strong repulsive forces above nuclear density, needs no further explanation. The time slice at $3 \mathrm{~ms}$ not only captures the launch of the electron neutrino burst, but also the interesting phase when the dynamical bounce-shock stalls (i.e., the postshock velocities become negative). At this time, long before any neutrino heating can take place, the infalling 
material does no longer change the direction of its velocity at the shock front. After deceleration at the accretion shock it continues to drift toward the center of the star. Puffed-up by the dissipation of the kinetic energy acquired during infall, however, the net volume of accumulated shock-heated material still increases such that the accretion front continues to expand to larger radii in a quasi-stationary manner. Only after the accretion front has reached a position farther out in the gravitational well, where less kinetic energy is dissipated in the shock, do the temperature difference between the receding hot neutrinospheres and the cooler postshock matter become favorable for neutrino heating. These conditions are not met before a time of $50 \mathrm{~ms}$ after bounce. In spherically symmetric simulations the accretion front reaches a maximum radius around $150 \mathrm{~km}$ at about $100 \mathrm{~ms}$ after bounce and recedes slowly thereafter. Therefore, we choose a third snapshot in our comparison at this late phase where neutrino cooling and heating influence the quasi-stationary evolution.

\subsection{Hydrodynamics}

As described above, the dynamical simulations are based on the two very different hydrodynamics codes AGILE and PROMETHEUS. In order to disentangle hydrodynamics differences from neutrino transport differences in our results it is helpful to perform a comparison of an adiabatic collapse where all neutrino interactions are suppressed. We found a simple test case that poses similar challenges to the hydrodynamics algorithms as the case with full transport. We take the progenitor model of run N13 and replace the electron fraction and entropy as a function of enclosed mass by the values obtained at bounce in the N13 run with full transport. By this measure, the Chandrasekhar mass at core bounce, which depends on electron fraction and temperature, is imposed already at the beginning of the simulation. As expected, the adiabatic collapse of this modified progenitor leads to bounce and shock formation at a similar mass coordinate as in the simulation with full transport, and therefore to similar conditions around bounce.

Figure 1 shows the situation at $3 \mathrm{~ms}$ after bounce. This is the critical time when the dynamical shock in the full models stalls to turn into an accretion front. We find very good agreement in the density and velocity profiles. The latter show the same timing and amplitude of reflected sound waves in the ringing neutron star. Also the entropy profiles agree well. The entropy profile contains information about the evolution of the shock strength because the otherwise conserved entropy of a fluid element can only change due to the dissipation of kinetic energy when matter passes through the shock front. The profile shows that both the shock in AGILE and the shock in PROMETHEUS start with a similar strength (almost identical entropy peak at $0.55 \mathrm{M}_{\odot}$ ). In the further evolution, however, the shock in AGILE decays slightly faster, producing lower postshock entropies. The weaker shock propagates more slowly with respect to the mass coordinate so that a small offset of the shock position becomes visible at $3 \mathrm{~ms}$ after bounce.

\subsection{Newtonian $13 M_{\odot}$ Model}

For the runs that include neutrino transport, we start the comparison with an investigation of differences in the

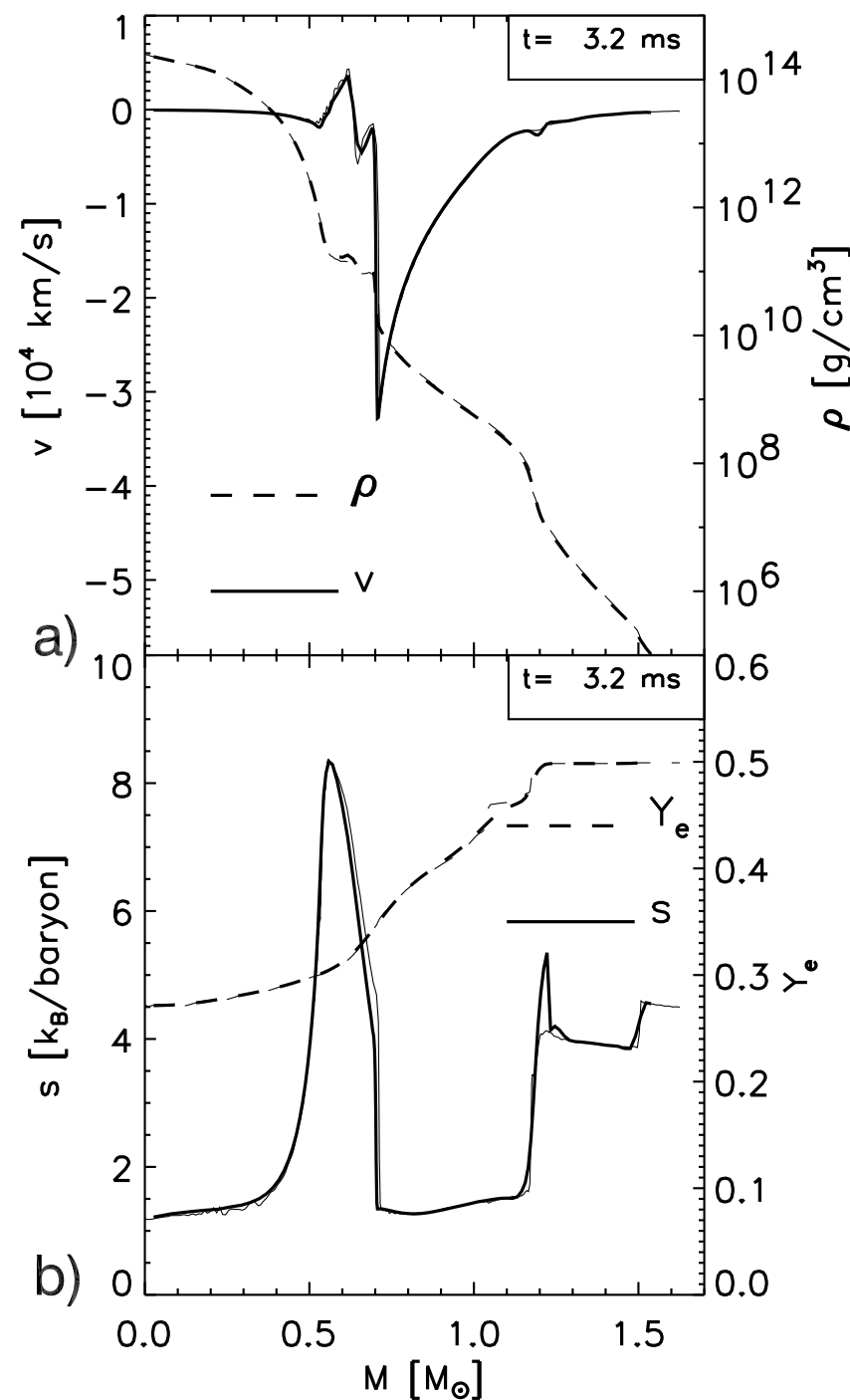

FIG. 1.- Snapshots at $3 \mathrm{~ms}$ after bounce for the adiabatic collapse of the modified N13 progenitor. Data from the AGILE simulation are drawn with thick lines. Data from the PROMETHEUs simulation are drawn with thin lines. Panel (a) shows the velocity (solid lines) and density (dashed lines) profiles, panel (b) the entropy (solid lines) and electron fraction (dashed lines). The hydrodynamics simulations agree well. The shock strength decays slightly faster in AGILE than in PROMETHEUS. The weaker shock in AGILE tends to propagate more slowly in mass and to produce a smaller postshock entropy.

N13 model, in which only $\nu_{e}$ and $\bar{\nu}_{e}$ are taken into account. Figure 2 presents the conditions at bounce. The neutrino luminosities are shown in panel (a). We find transient differences of order $30 \%$ in the electron neutrino luminosities at bounce in the diffusive regime inside of the nascent shock. The luminosities are in good agreement outside of the shock front and the electron neutrino rms energies in panel (c) agree well in all domains. The electron antineutrino rms energies are very uncertain at this time because the antineutrino abundance is negligi- 

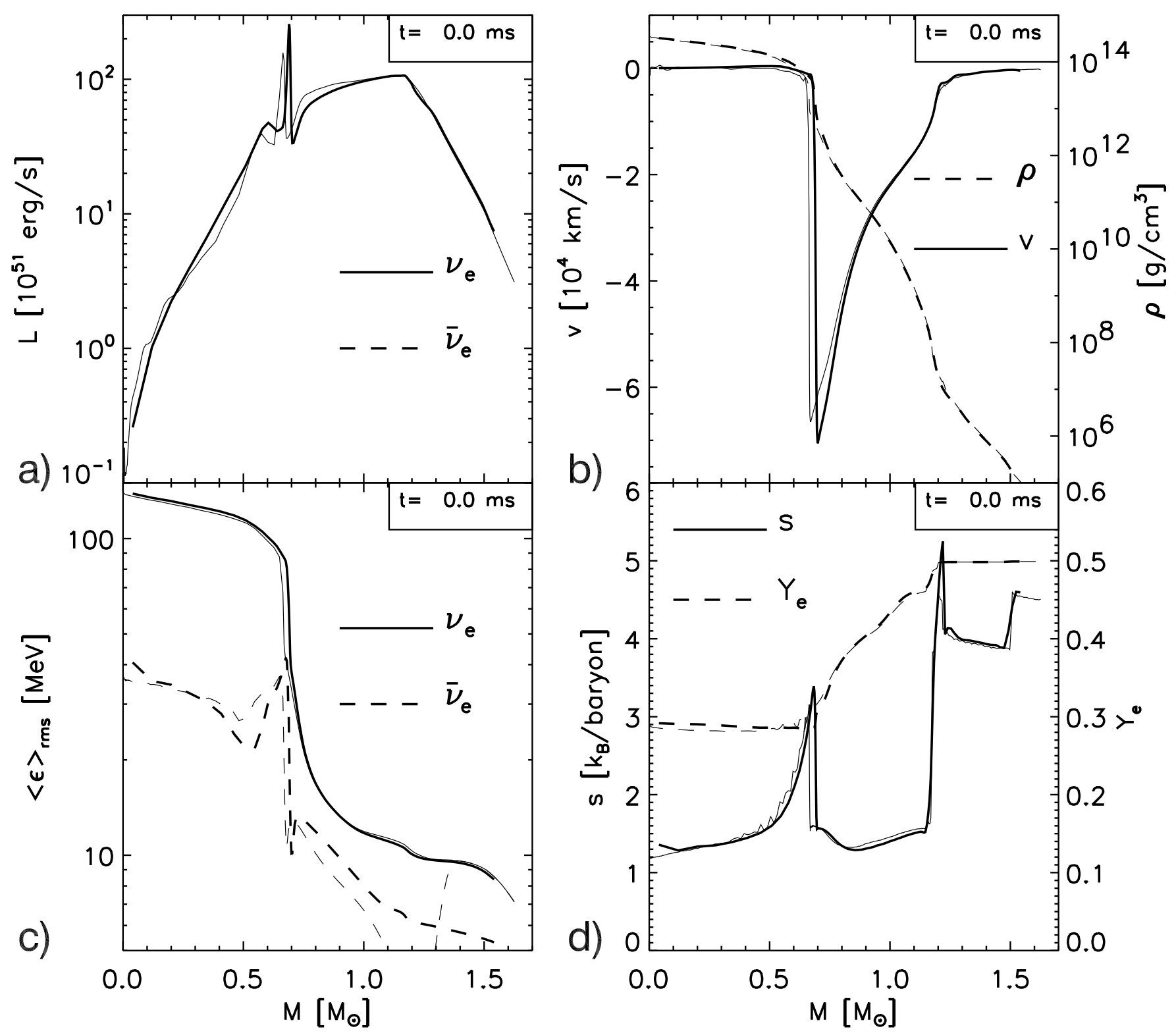

Fig. 2.- Snapshots at bounce for model N13. Data from the AGILE-BOLTZTRAn simulation are drawn with thick lines. Data from the VERTEX simulation are drawn with thin lines. Panel (b) shows the velocity (solid lines) and density (dashed lines) profiles, panel (d) the entropy (solid lines) and electron fraction (dashed lines). The neutrino luminosities are given in panel (a) and the neutrino rms energies in panel (c). Solid lines refer to electron neutrinos and dashed lines to electron antineutrinos. The central electron fraction in vERTEX is smaller than in AGILE-BOLTZTRAN and the shock forms at a slightly smaller enclosed mass. The high electron degeneracy during collapse suppresses electron antineutrino production so that the corresponding luminosity at bounce is below the threshold of panel (a).

ble under the electron-degenerate conditions at bounce. Important quantities at bounce are the entropy and the electron fraction in panel $(\mathrm{d})$ because they determine the size of the causally connected homologous core. A shock forms when the outgoing pressure wave from the bounce at nuclear densities reaches its edge. The differences in the electron fraction are of order $3 \%$, the differences in the neutrino abundances are even smaller.
Most of these differences are introduced during the last $2 \mathrm{~ms}$ before bounce. The profiles are in nearly perfect agreement before. This becomes evident in panels (c) and (d) of Fig. 3 where we plot the entropy and lepton fractions of the innermost zone as functions of density during core collapse. We find that the differences in deleptonization appear just before neutrino trapping sets in, i.e. when the effective electron capture rates are 
highest. The entropy evolution shows perfect agreement during infall, but after neutrino trapping, an unphysical entropy increase in the innermost zones takes place in the AGILE-BOLTZTRAN simulation. Fig. 2] clearly demonstrates that this entropy increase only occurs in the innermost zone. Probably more significant is the small deviation of order $5 \%$ in the lepton and electron fraction which appears at the same time. It is not confined to the innermost zone and influences the enclosed mass at shock formation.

The formation of the shock is visible in the velocity profiles in panel (b) of Fig. 2 The difference in enclosed mass $\sim 3 \%$ between AGILE-BOLTZTRAN and VERTEX is qualitatively consistent with the differences in the electron fraction profiles in Fig. 2 d. The infall velocities in the outer core agree well. Panel (b) also shows the density profiles of the N13 run at bounce. We conclude the discussion of bounce with the observation that there are small visible differences between the two N13 simulations, but none of them is likely to induce large deviations in the postbounce evolution.

Figure 3 shows the mass trajectories for both runs during the first $10 \mathrm{~ms}$ after bounce, in panel (a) for AGILEBOLTZTRAN and in panel (b) for VERTEX. The rising dashed line marks the shock position. A first inspection reveals a difference of $15 \%$ in shock radius at $7 \mathrm{~ms}$ after bounce. As we will see later in Fig. [5] this difference is transient. It appears after a short dynamical phase of shock propagation, long before any neutrino heating takes place and long before the accretion front has reached its maximum radius at $\sim 250 \mathrm{~km}$ in this optimistic model with reduced input physics.

The discrepancy originates from a different shock strength as cause and a different neutrino burst behavior as consequence and amplification mechanism. The gradients of the mass trajectories in Figs. 3 a and b indicate the velocity of the material in the postshock region (at the right hand side of the thick dashed line that represents the position of the shock). The bounce-shock is dynamical at the beginning and drives matter outward with positive postshock velocities. At about $4 \mathrm{~ms}$ after bounce, the shock stalls because of the nuclear dissociation of infalling matter and neutrino losses. It converts into an accretion shock, characterized by jump conditions that connect the high speed/low density infalling material to the low speed/high density postshock material. There is an important difference between the dynamical and the accretion shock: The postshock material behind a dynamical shock is diluted between the rarefaction wave and the shock front, while the material behind an accretion front continues to be compressed due to the accumulated mass. The examination of the mass trajectories in Fig. 3ab indicates that VERTEX maintains a dynamical shock for a longer time than AGILE-BOLTZTRAN. This is consistent with the result of the hydrodynamics comparison in Fig. 1

This difference in shock propagation is initially not very large. It is significantly amplified by the coincidence of the electron neutrino burst with the transition from a dynamical to an accretion shock. As the shock compresses the infalling lepton-rich material, the fermionic electrons have to populate high energy levels and are rapidly converted to neutrinos by captures on protons as soon as the density is low enough for the produced neu- trinos to escape. This neutrino burst can be launched by the shock while it is still in its dynamical phase or after it has stalled to an accretion front. If the neutrino burst is launched during the dynamical phase, infalling matter deleptonizes due to the immediate compression in the shock front. After that, electron captures are reduced quickly because the matter re-expands behind the dynamical shock and the density drops again. In an accretion shock, the infalling matter experiences the same initial deleptonization in the shock front, but continues to emit neutrinos due to the compression behind the shock. The neutrino burst from an accretion shock is therefore more intense. The neutrino emission behind the shock removes energy and lepton number and reduces the pressure support. It accelerates the attenuation of the weaker shock and thereby amplifies the initial difference in shock strength. Using the lepton number source term, $q_{\ell}$, in units of generated leptons per baryon and second, we shaded in panels (a) and (b) of Fig. 3 the regions where the neutrino emission $4 \pi r^{2} \rho / m_{B} \times q_{\ell}$ exceeds the thresholds of $10^{51} \mathrm{~cm}^{-1} \mathrm{~s}^{-1}, 2 \times 10^{51} \mathrm{~cm}^{-1} \mathrm{~s}^{-1}$, and $3 \times 10^{51} \mathrm{~cm}^{-1} \mathrm{~s}^{-1}$. The comparison of panels (a) and (b) illustrates that more neutrinos are lost from the region behind the weaker shock in the AGILE-BOLTZTRAN simulation. The initially stronger shock in the VERTEX simulation suffers less neutrino losses and leads to a more rapidly expanding accretion front.

After this investigation we can easily interpret the time slice at $3 \mathrm{~ms}$ after bounce presented in Fig. 4. In the luminosity, panel (a), we see good agreement during the launch of the neutrino burst. The somewhat higher rms energies of the burst electron neutrinos, compared to the previously emitted ones, are visible in panel (c). The entropy profile in panel (d) shows a slightly weaker shock in AGILE-BOLTZTRAN than in VERTEX, very similar to the differences found for the hydrodynamics comparison in Fig. 1] The electron fraction profiles now show that the deleptonization of the postshock region between 0.8 and $1.0 \mathrm{M}_{\odot}$ is more pronounced in AGILE-BOLTZTRAN. This is consistent with the higher density visible in panel (b). The positive velocities behind the shock also demonstrate that the VERTEX shock has still a larger kinetic energy in this snapshot. The shock in VERTEX will stall somewhat later than in AGILE-BOLTZTRAN.

The further evolution is best followed in the timedependent diagrams in Fig. 5. In panel (b), the luminosities and rms energies are shown, sampled at a fixed radius of $500 \mathrm{~km}$. The two neutrino signals are qualitatively very similar. The neutrino burst in AGILE-BOLTZTRAN is somewhat broader than in VERTEX and has a $7 \%$ smaller peak luminosity. The deviations after the burst are at most $15 \%$ around $50 \mathrm{~ms}$ after bounce. This difference is a late consequence of the deleptonization differences during the neutrino burst. The material in VERTEX is left with higher electron fraction and higher entropy after the neutrino burst. Hence it deleptonizes at a higher rate afterwards. The rms energies tend to be lower in VERTEX than in AGILE-BOLTZTRAn. Finally, panel (a) compares the accretion front trajectories over a longer period of time. We find the described differences in the early expansion of the accretion front. At $85 \mathrm{~ms}$ after bounce, however, the trajectories cross and a larger maximum radius is reached in the AGILE-BOLTZTRAN simulation. The maximum radius of the accretion front differs 

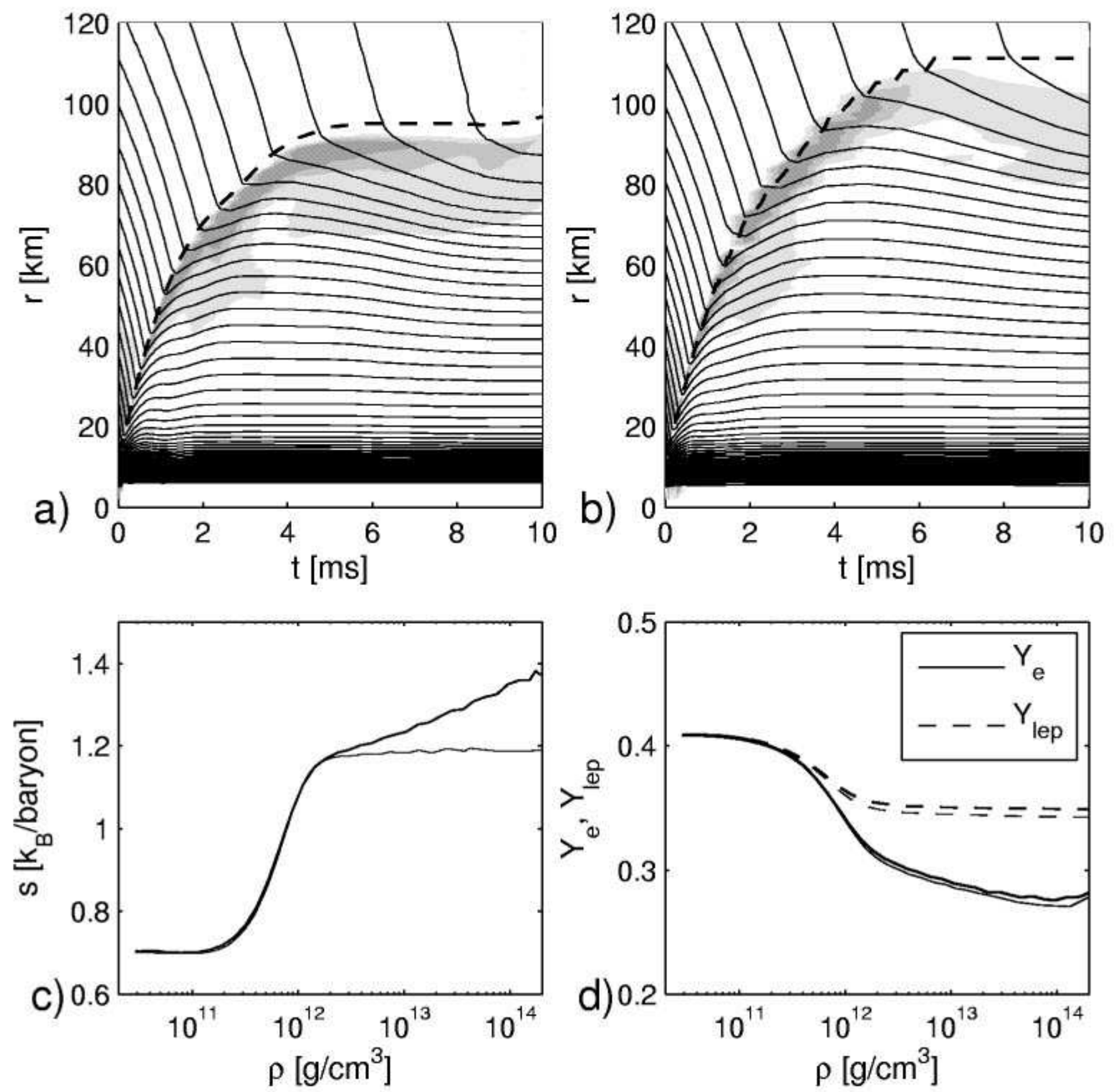

Fig. 3.- Panel (a) shows the first $10 \mathrm{~ms}$ after bounce for model N13 in the simulation with AGILE-BOLtZTRAn. Panel (b) shows the same time period in the simulation with VERTEX. The thin lines represent trajectories of fluid elements spaced with an interval of 0.02 $\mathrm{M}_{\odot}$. The dashed line marks the shock position as a function of time. The gradients of the mass trajectories at the right hand side of the dashed line indicate the postshock velocities. At $\sim 4 \mathrm{~ms}$ after bounce the shock has stalled. It turns into an expanding accretion front with negative postshock velocities. Areas with strong neutrino emission are shaded in three levels corresponding to values of one, two, and three times $10^{51}$ neutrinos per centimeter and second (i.e. for $4 \pi r^{2} \rho / m_{B} \times q_{\ell}$, where $q_{\ell}$ is the lepton number source term in units of leptons per baryon and second). The coincidence of the launch of the neutrino burst with the transition from a dynamical to an accretion shock in this model leads to an amplification of the small hydrodynamics differences found above. The region behind the weaker shock in AGILE-BOLTZTRAN experiences more compression. It therefore deleptonizes more rapidly and the weak shock loses even more pressure support than the stronger shock in the VERTEX simulation. The accretion front therefore expands more slowly in the AGILE-BOLTZTRAN simulation. Panel (c) compares the entropy of the innermost zone as a function of density in AGILE-BOLTZTRAN (thick line) and in VERTEX (thin line). The agreement before trapping is close to perfect, dynamically insignificant differences appear at larger densities. Panel (d) shows an analogous comparison for the electron fraction (solid lines) and lepton fraction (dashed lines) in the innermost zone. 

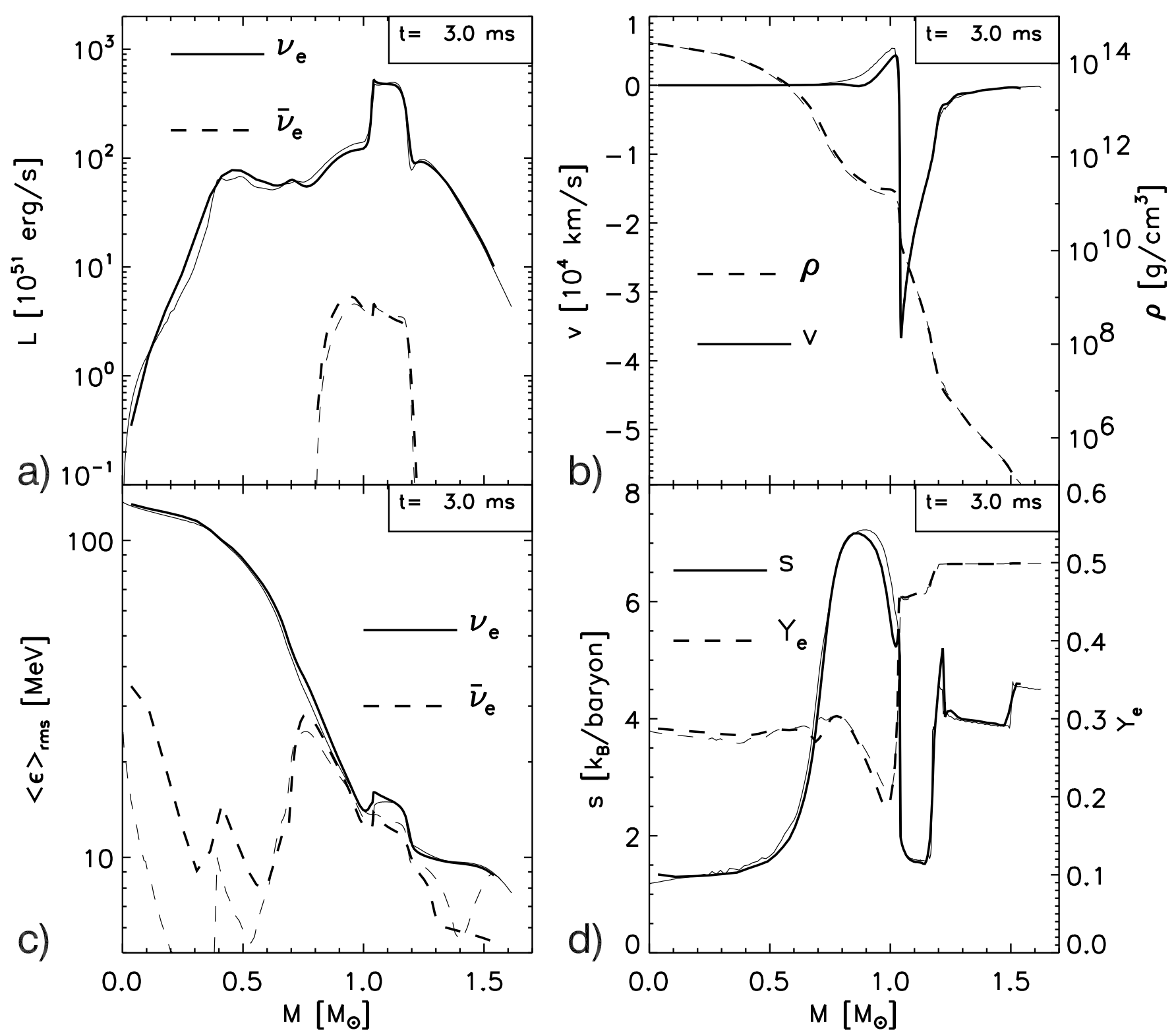

Fig. 4.- Snapshots at $3 \mathrm{~ms}$ after bounce for model N13. Data from the AGILE-BoltzTran simulation are drawn with thick lines. Data from the VERTEX simulation are drawn with thin lines. Panel (b) shows the velocity (solid lines) and density (dashed lines) profiles, panel (d) the entropy (solid lines) and electron fraction (dashed lines). Panels (a) and (c) show the neutrino luminosities and rms energies respectively. Solid lines refer to electron neutrinos and dashed lines to electron antineutrinos. The snapshot reveals similar small differences as the hydrodynamics comparison in Fig. 1] 

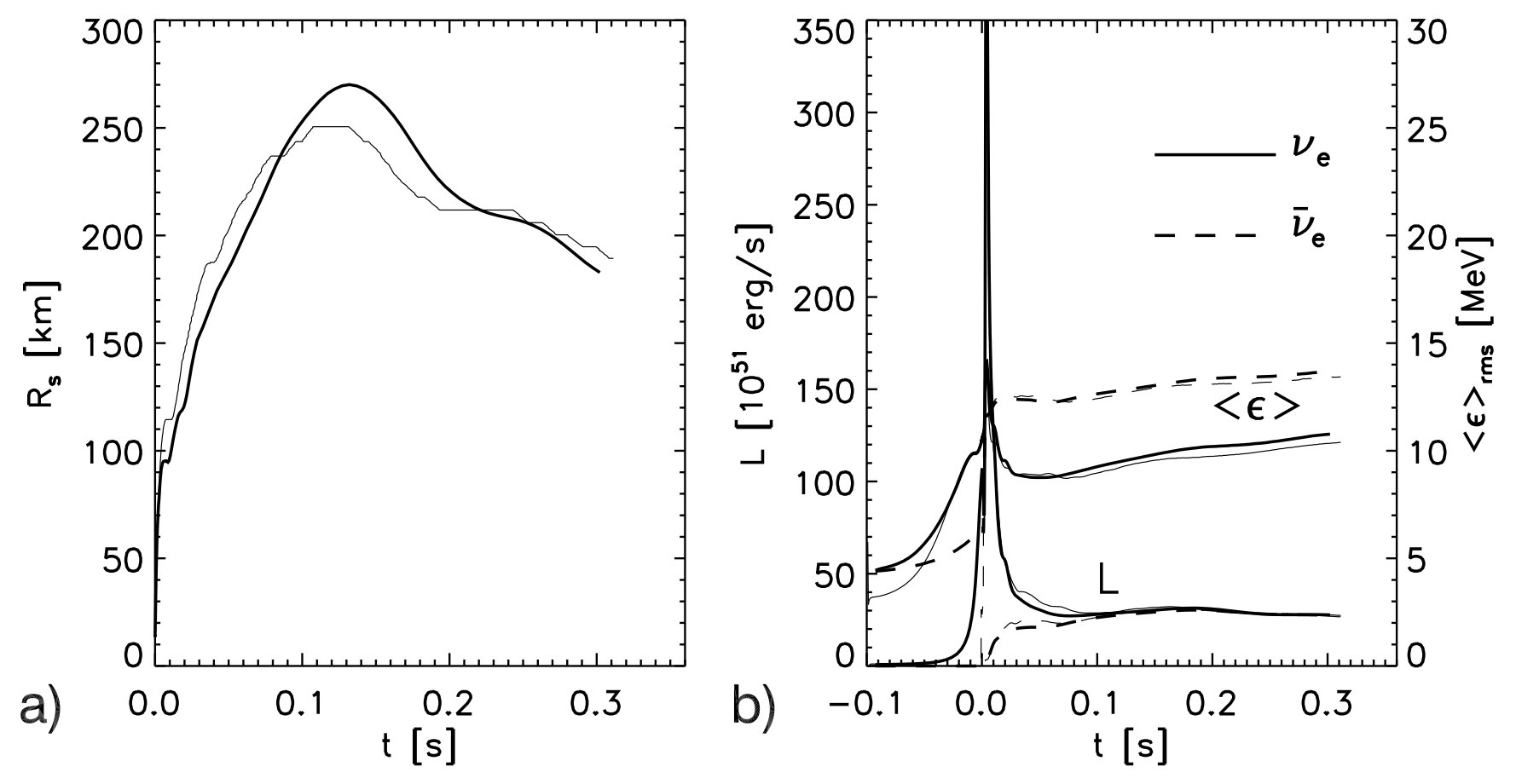

FIG. 5.- The shock position as a function of time for model N13 is shown in panel (a). The shock in VERTEX (thin line) propagates initially faster and nicely converges after its maximum expansion to the position of the shock in AGILE-BOLTZTRAN (thick line). The neutrino luminosities and rms energies for model N13 are presented as functions of time in panel (b). The values are sampled at a radius of 500 $\mathrm{km}$ in the comoving frame. The solid lines belong to electron neutrinos and the dashed lines to electron antineutrinos. The line width distinguishes between the results from AGILE-BOLTZTRAN and VERTEX in the same way as in panel (a). The luminosity peaks are nearly identical, the rms energies have the tendency to be larger in AGILE-BOLTZTRAN.

by $\sim 8 \%$ between the two simulations. This difference is due to the higher preshock entropies in the AGILEBOLTZTRAN simulation visible in Fig. 6 $\mathrm{d}$. The difference in the entropies of the infalling material stems from the interface between the silicon layer and the material in nuclear statistical equilibrium (NSE). The burning in AGILE-BOLTZTRAN cannot be switched off completely for this comparison, because conversions between non-NSE and NSE are unavoidable when the adaptive grid moves its zones relative to the mass coordinate. This produces a local entropy peak at the composition interface (see Fig. $4 \mathrm{~d}$ at an enclosed mass of $1.2 \mathrm{M}_{\odot}$ ) which crosses the accretion front at $85 \mathrm{~ms}$ after bounce. The higher entropy leads to a temporarily lowered accretion rate which allows the accretion front in the AGILE-BOLTZTRAN simulation to propagate to a larger radius than in the VERTEX simulation where no conversions between non-NSE and NSE occur during this simulation with reduced input physics. After the initial expansion phase, where matter piles up on the neutron star hydrostatically, the pressure support in the cooling region starts to diminish rapidly and the matter in the heating region is pulled inward from below (see e.g. Janka (2001); Liebendörfer et al. (2001)). Simultaneously, the mass shell in the preshock region containing the described entropy differences has fallen through the shock and the entropy becomes more similar again. Therefore the trajectories of the accretion front converge and agree well during the shock recession phase.

We finish the comparison of the N13 model with a closer look at a time slice at $150 \mathrm{~ms}$ after bounce (Fig.
6), which is a snapshot during this quasi-stationary accretion phase. Panel (a) demonstrates excellent agreement of the luminosities in all regions of the computational domain. The rms energies in panel (c) show small differences, especially outside of $100 \mathrm{~km}$ radius. The velocity profiles in panel (b) agree well if one disregards the different shock positions explained above. The higher accretion rates in VERTEX of material with lower entropy lead to a higher density in the cooling region. This is visible in the density profiles in panel (b) and the entropy profiles in panel (d). The reaction time scale is comparable to the dynamical time scale inside the gain radius at $115-120 \mathrm{~km}$. The infalling fluid element therefore is close to weak equilibrium in the given neutrino background. Since the neutrino luminosities are very similar in the two simulations, the larger density of the VERTEX run requires a lower equilibrium electron fraction in the cooling region inside the gain radius. The corresponding differences in the pressure profiles imply less support for the shock and are consistent with a smaller radius of the accretion front.

\subsection{General Relativistic $15 M_{\odot}$ Model}

For the analysis of the G15 simulations we start again with the description of the conditions at bounce. The evolution of the entropy and lepton fraction in the innermost zone is shown in panels (c) and (d) of Fig. 7 Compared to model N13 with Newtonian gravity, the central entropy in the general relativistic G15 model is $15 \%$ higher and the lepton and electron fractions are $5 \%$ lower. The deviations between the VERTEX and AGILE- 

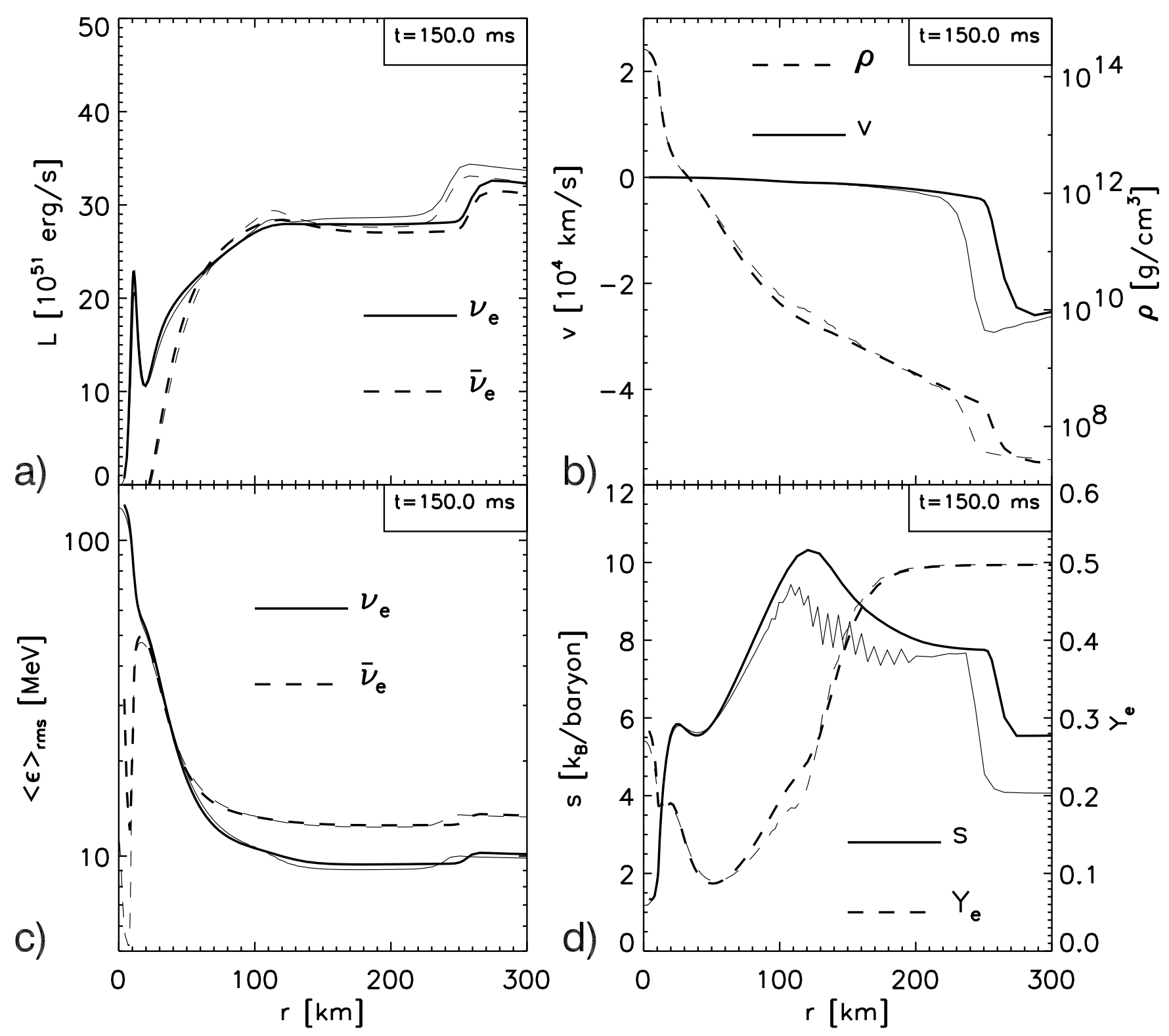

FIG. 6.- Snapshots at $150 \mathrm{~ms}$ after bounce for model N13. Data from the AGILE-BOLTZTRAN simulation are drawn with thick lines. Data from the VERTEX simulation are drawn with thin lines. Panel (b) shows the velocity (solid lines) and density (dashed lines) profiles, panel (d) the entropy (solid lines) and electron fraction (dashed lines). The neutrino luminosities and rms energies are shown in panels (a) and (c) respectively. Solid lines refer to electron neutrinos and dashed lines to electron antineutrinos. This stationary-state situation is typical of the neutrino heating phase at later time after bounce. The agreement is satisfactorily close in most quantities.

BOLTZTRAN simulations are of order $3 \%$ with the exception of an entropy increase in the innermost zone in the AGILE-BOLTZTRAN simulation. As shown in Fig. 8], excellent agreement is found in all other regions of the model up to the burning front, where different approximations in tracking the composition and nuclear burning explain the larger differences. In contrast to the entropy difference in the innermost zone, the small differences in the electron fraction apply to the whole high-density region enclosed by the shock.

The luminosity profiles at bounce are displayed in Fig. 87. Outside of the shock front, they have been set during collapse and agree well. Also the luminosities in the diffusive regime reveal no mentionable differences. As a consequence of the differences of the electron fraction visible in panel (d), the velocity and density profiles in panel (b) show the formation of the shock front in VERTEX at a slightly deeper point than in AGILE-BOLTZTRAN. In con- 

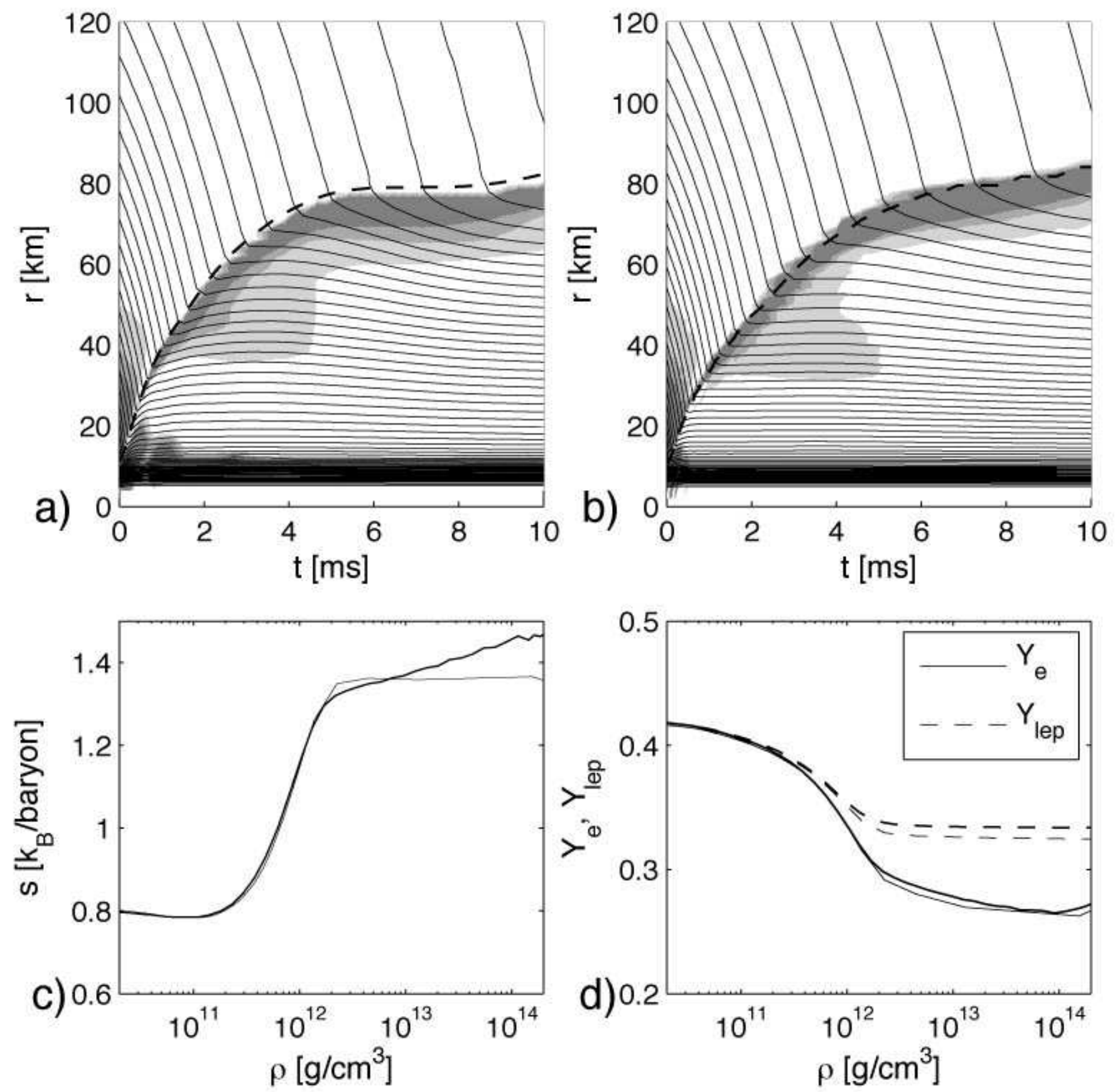

Fig. 7.- Panel (a) shows the first $10 \mathrm{~ms}$ after bounce for model G15 in the simulation with AGILE-BOLtzTran. Panel (b) shows the same time period in the simulation with VERTEX. The thin lines represent trajectories of fluid elements spaced with an interval of 0.02 $\mathrm{M}_{\odot}$. Areas with strong neutrino emission are shaded in three levels corresponding to values of one, two, and three times $10^{51}$ neutrinos per centimeter and second (i.e. for $4 \pi r^{2} \rho / m_{B} \times q_{\ell}$, where $q_{\ell}$ is the lepton number source term in units of leptons per baryon and second). Both codes obtain an extended region of strong neutrino emission behind the shock, which turns into an accretion front (dashed line) at 3-4 ms after bounce. Panel (c) compares the entropy of the innermost zone as a function of density in AGILE-BOLTZTRAN (thick line) and in VERTEX (thin line). The agreement before trapping is close to perfect, dynamically insignificant differences appear at larger densities. Panel (d) shows a comparison of the electron fraction (solid lines) and lepton fraction (dashed lines) in the innermost zone. The deviations between the two codes are of order $3 \%$.

sideration of this displacement in the shock position, also the rms neutrino energies in panel (c) are in satisfactory agreement.

The early postbounce evolution of the G15 model is less sensitive to small differences than the previously discussed N13 simulation. The main reason is the weaker bounce shock. General relativis- tic effects during core collapse shift the sonic point to a $20 \%$ smaller enclosed mass and lead to a less energetic bounce shock (Liebendörfer et al. 2001; Bruenn. DeNisco. \& Mezzacappa 2001) which has to dissociate more infalling material. The inclusion of $\mu$ and $\tau$-neutrinos in the G15 runs causes additional energy drain from the region behind the shock. After the 

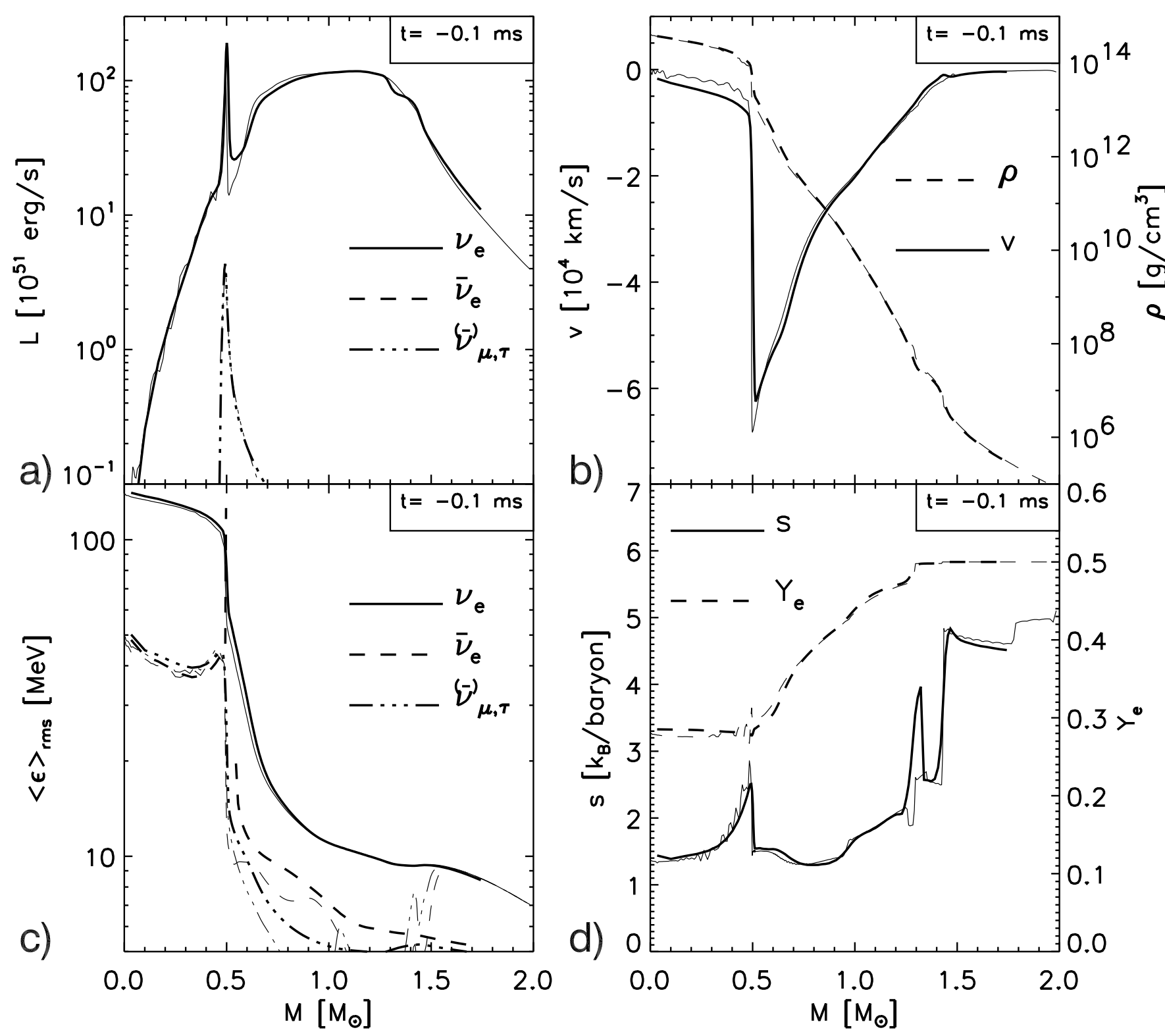

FIG. 8.- Snapshots at bounce for model G15. Data from the AGILE-BOLTZTRAN simulation are drawn with thick lines. Data from the VERTEx simulation are drawn with thin lines. Panel (b) shows the velocity (solid lines) and density (dashed lines) profiles, panel (d) the entropy (solid lines) and electron fraction (dashed lines). The neutrino luminosities and rms energies are given in panels (a) and (c), respectively. Solid lines correspond to electron neutrinos, dashed lines to electron antineutrinos, and dash-dotted lines to $\mu$ - or $\tau$-neutrinos (or their antiparticles). In the region enclosed by the shock the luminosities in AGILE-BOLTZTRAN are smaller than in VERTEX, consistent with the larger central electron fraction and a slightly larger enclosed mass at shock formation.

shock has stalled within $1 \mathrm{~ms}$ in both G15 simulations, the electron neutrino burst is launched during the accretion shock phase. As expected from the discussion of the neutrino emission from the shock in the N13 model, panels (a) and (b) in Fig. 7 reveal a well extended region of high neutrino emission behind the shock. Since the postshock matter develops negative velocities a few milliseconds after bounce, the further evolution is deter- mined by the continued accumulation of accreted matter, more and more effectively cooled as the accretion front reaches layers with lower matter densities and neutrino opacities. This quasi-stationary evolution is less sensitive to differences in the numerics or input physics than the dynamical shock propagation in the more optimistic N13 simulation. The feedback between neutrino transport and hydrodynamics amplifies differences in the 
propagation of dynamical shocks, because the material behind the weaker shock emits more neutrinos so that the shock loses even more pressure support. In case of a quasi-stationary accretion front, differences are likely to be reduced because a larger accretion rate produces larger neutrino losses.

However, differences can still be observed: The AGILEBOLTZTRAN shock is stronger at formation. As in the N13 simulations, this is partly due to the higher electron fraction in the homologous core of the AGILE-BOLTZTRAN simulation. A difference in the entropy profiles is left behind when the shock passes an enclosed mass of 0.75 $\mathrm{M}_{\odot}$ in Fig. 91. This points to a larger deviation between the initial shock strengths than in the N13 simulations so that the AGILE-BOLTZTRAN shock expands initially faster in Fig. 7 . The differences between the electron fraction profiles in Fig. 9 $\mathrm{d}$, however, suggest that later on a faster deleptonization in AGILE-BOLTZTRAN damps the expansion of the accretion front and as a consequence the shock positions in both simulations converge again. This connection between a somewhat enhanced neutrino loss and a deceleration of the expansion of the accretion front is supported by the shaded areas in panels (a) and (b) of Fig. 7 which highlight differences in the regions of strong neutrino emission in both simulations.

In Fig. 9p, a lower density at $0.75 \mathrm{M}_{\odot}$ is caused by the higher entropy in the AGILE-BOLTZTRAN run. The velocity profiles at $3 \mathrm{~ms}$ after bounce are in very good agreement. A slightly higher infall velocity in front of the shock leads to a positive entropy gradient between $0.8 \mathrm{M}_{\odot}$ and $\sim 1 \mathrm{M}_{\odot}$ in VERTEx. Also the neutrino luminosities at $3 \mathrm{~ms}$ after bounce in Fig. 97 do not reveal new features, except for the presence of $\mu$ - and $\tau$-neutrinos that had not been included in the N13 run. Because of the absence of charged-current reactions of these neutrinos, they decouple at higher densities and reach appreciable luminosities earlier in the evolution, before the appearance of electron antineutrinos, which are initially suppressed by the high electron degeneracy. The apparent difference in the electron antineutrino luminosity between AGILE-BOLTZTRAN and VERTEX is due to a small time lag in displaying this rapidly rising quantity.

The further evolution is resumed in Fig. [10. Panel (a) compares the position of the accretion front as a function of time for the G15 models. During the expansion of the accretion front, we find very good agreement and the maximum radius is nearly identical. Afterwards, the accretion front retreats somewhat more slowly in the AGILE-BOLTZTRAN simulation than in the VERTEX simulation. In the latter, the retraction transiently stagnates between 150 and $180 \mathrm{~ms}$ after bounce. Such features are caused by the steep density drop at the infalling interfaces between layers of different composition outside of the iron core. The transition to the oxygen-rich silicon layer passes the shock at about $165 \mathrm{~ms}$ after bounce. AGILE-BOLTZTRAN tracks the structure of the outer layers less accurately because of the artificial diffusion introduced by the adaptive grid. Discrete transitions between layers are therefore washed out to some extent such that their impact on the trajectory of the accretion front is less pronounced, although still qualitatively visible. The luminosity peaks during the electron neutrino burst deviate only by $3 \%$ in the G15 simulations, the average peak value is $3.8 \times 10^{53} \mathrm{erg} / \mathrm{s}$ with a half-width of $6 \mathrm{~ms}$.
The further time evolution of the luminosities and rms energies in panel (b) reveals $\sim 20 \%$ larger values in the VERTEX run. These are at least in part a consequence of the increased accretion rate in the VERTEX simulation during the retraction phase of the accretion front. We will make this argument more precise in the following discussion of late time slices.

We present two time slices for the long-term evolution of the G15 simulation. The first time slice is at $100 \mathrm{~ms}$ after bounce when the neutrino heating is most efficient. The second time slice at $250 \mathrm{~ms}$ marks the end of the time period covered by the simulations. The time slice at $100 \mathrm{~ms}$ is given in Fig. 11] Panel (b) shows the shock in VERTEX at a slightly smaller radius and the preshock infall velocities to be somewhat higher than in AGILEBoltzTran. The luminosities in panel (a) are up to $20 \%$ larger in VERTEX. The luminosity discontinuity across the shock front caused by the Doppler frequency shift and angular aberration for an observer in the comoving frame is also larger in VERTEX because of the larger luminosity and the larger velocity jump visible in panel (b). Otherwise the relative differences between the two runs are just inverse to the situation we analyzed in Fig. 6] in case of the N13 simulation. Now AGILE-BOLTZTRAN has a higher density in the shocked material in panel (b) and a correspondingly lower entropy and electron fraction in panel (d). Also the neutrino rms energies in panel (c) are now lower than in the VERTEX simulation.

We also include the latest time slice at $250 \mathrm{~ms}$ after bounce in Fig. 12 The panels show the same qualitative features we have discussed in the context of Fig. 11] but at this late time to a much larger extent. The protoneutron star in the VERTEX simulation is more compact, causing the accretion front to reside at a smaller radius and the luminosities of all neutrino flavors to be larger and to have harder spectra than in the AGILEBOLTZTRAN simulation. Due to the smaller radius of the accretion front and higher infall velocities ahead of it, the postshock entropy is significantly higher in the VERTEX run.

A more compact neutron star with a larger mass and a stronger gravitational potential (which would lead to higher preshock velocities) can be a consequence of a higher mass accretion rate. Indeed we observe differences in the mass flux to the shock, which can be caused by the different quality to follow structures in the outer stellar layers in both simulations. An infalling density feature in VERTEX (which might evolve differently due to the different treatment of "burning" and thus different entropy and pressure, or may be smoothed by the diffusivity of the adaptive grid in AGILE-BOLTZTRAN) transiently enhances or reduces the accretion rate. When, for example, the transition to the oxygen-rich silicon layer falls in shortly before $200 \mathrm{~ms}$ after bounce, the accretion rate decreases sharply and the retraction of the accretion front in the VERTEX simulation stagnates. This is consistent with the luminosity reduction during this phase. But the artificial diffusion in the outer layers of the AGILEBOLTZTRAN simulation can explain only transient differences of the mass accretion rate and of the total accreted mass, because an associated redistribution of matter and a modification of the preshock structure is limited to a certain radial domain. It should, however, not produce persistent differences in the density distribution behind 

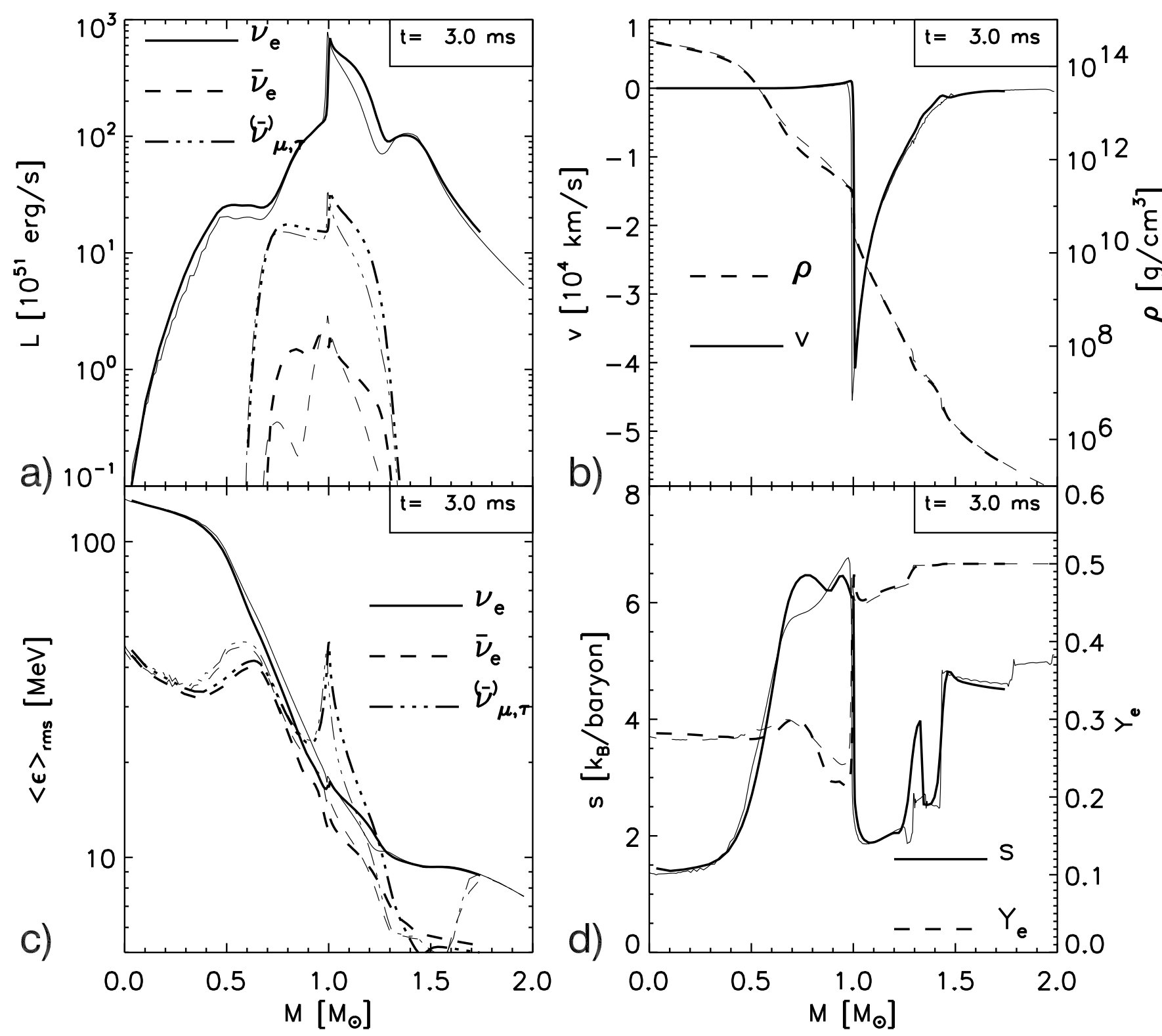

FIG. 9.- Snapshots at $3 \mathrm{~ms}$ after bounce for model G15. Data from the AGILE-BOLTZTRAN simulation are drawn with thick lines. Data from the VERTEX simulation are drawn with thin lines. Panel (b) shows the velocity (solid lines) and density (dashed lines) profiles, panel (d) the entropy (solid lines) and electron fraction (dashed lines). The neutrino luminosities and rms energies are given in panels (a) and (c), respectively. Solid lines correspond to electron neutrinos, dashed lines to electron antineutrinos, and dash-dotted lines to $\mu$ - or $\tau$-neutrinos (or their antiparticles). Variations in the entropy profiles reflect the differences in the shock strength at bounce. The deleptonization by the neutrino burst occurs an instant earlier in the AGILE-BOLTZTRAN simulation.

the shock, which in fact can be seen in Figs. 111 and 12 (cf. the density profiles in panels (b)). A closer inspection of our models in fact reveals that the mass accretion rate outside of the shock and the baryonic mass accumulated in the neutron star show temporary differences only between $\sim 30 \mathrm{~ms}$ and $\sim 200 \mathrm{~ms}$, but become very similar again toward the end of our simulations.

The systematically evolving and growing difference during the long-term evolution must therefore be caused by another effect. The combination of higher luminosities, higher rms energies and higher entropies at the neutrinosphere reminds us of the differences found between Newtonian and general relativistic simulations, where they are due to differences between the Newtonian and relativistic gravitational potential (Bruenn. DeNisco. \& Mezzacappa 2001; Liebendörfer et al. 2001). Both of our numerical methods are designed to accurately describe the hydrostatic 

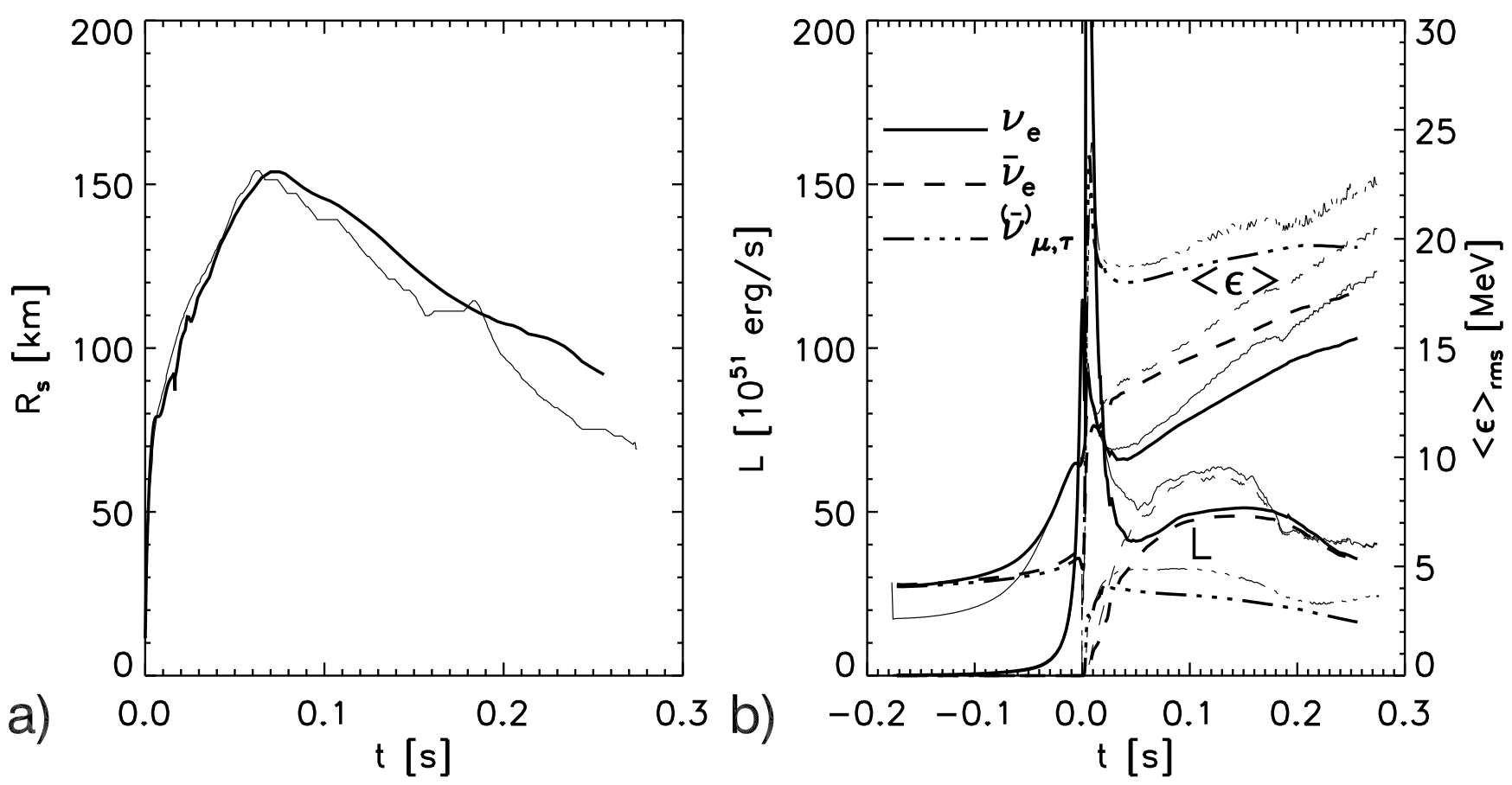

FIG. 10. - Panel (a) shows the position of the accretion front as a function of time for model G15. The two simulations predict a very similar early expansion of the accretion front up to a maximum radius of $150 \mathrm{~km}$. Then, the accretion front in VERTEX (thin line) retreats faster than in AGILE-BOLTZTRAN (thick line) in response to the slightly stronger contraction of the nascent neutron star due to the approximate treatment of general relativity in the VERTEX simulation. The hump in the VERTEX shock position at $\sim 180$ ms after bounce corresponds to an entropy and density discontinuity at the bottom of the oxygen-rich silicon shell. Because the VERTEX simulation resolves the structure of the outer layers of the progenitor star more accurately than the diffusive adaptive grid in AGILE-BOLTZTRAN, this feature is less pronounced in the latter simulation. The neutrino luminosities and rms energies are presented as functions of time in panel (b). The values are sampled at a radius of $500 \mathrm{~km}$ in the comoving frame. The solid lines correspond to electron neutrinos, the dashed lines to electron antineutrinos, and the dash-dotted lines to $\mu$ - or $\tau$-neutrinos (or their antiparticles). The line width distinguishes between the results from AGILE-BOLTZTRAN (thick lines) and VERTEX (thin lines). The differences in the neutrino results are mainly-but not exclusively-indirect consequences (due to the more compact neutron star) of the approximate treatment of general relativity in the VERTEX simulation.

structure of the protoneutron star according to the solution of the Tolman-Oppenheimer-Volkoff (TOV) equation. But in the relativistic case differences in the potential can not only be caused by a difference of the enclosed mass at a given radius. In contrast to the Newtonian case, the relativistic potential depends highly nonlinearly on the structure of the configuration through its dependence on the mass distribution, pressure, and energy density. The gravitational potential is therefore sensitive to differences in the early post-bounce dynamics of the propagating shock (e.g., due to the different initial shock strength) and to pressure and entropy differences created at later times, e.g., associated with the transient differences of the mass accretion rate or due to the higher infall velocities ahead of the shock in the VERTEX run. The overestimation of the velocities in the collapse layer is also a consequence of the approximation of general relativity in case of VERTEX. The latter code uses only a gravitational potential that is corrected for general relativistic effects, but ignores relativistic kinematics. When the preshock values of the infall velocities reach 10-15\% of the speed of light, the velocities computed by VERTEX are overestimated in comparison to the relativistic velocities calculated by AGILE-BOLTZTRAN. This again has an influence on the long-term post-bounce evolution and thus feeds back into the core structure and causes a nonlinear response of the relativistic potential. While we find that the maximum densities at bounce and the following relaxation to a static situation are in good agreement between the VERTEX and AGILE-BOLTZTRAN runs, we subsequently observe clear deviations of the central density and of the density profile which gradually evolve and grow at later times. The VERTEX simulation develops a higher central density and a steeper density gradient outside of the high-density core, and thus a more compact neutron star with a higher relativistic potential. This is confirmed by Fig. 13 which shows the profiles of the metric coefficients $\alpha=g_{t t}^{1 / 2}$ and $\Gamma=g_{a a}^{-1 / 2} \partial r / \partial a$ in Eq. (11) at $250 \mathrm{~ms}$ after bounce. The smaller deviations from unity of the metric coefficients in the AGILE-BOLTZTRAN run are consistent with the less compact structure of the protoneutron star in this simulation. The profiles also show that the metric coefficients are nearly unity outside of the accretion front. The larger preshock velocities for the VERTEX run in Figs. 11] and 12p are therefore not a consequence of the fact that the lapse function is not included in the velocity plotted for this simulation. They are more likely caused by the disregard of kinematical effects and a stronger gravitational potential in the approximation of general relativity used in VERTEX.

\subsection{Discussion}

This work extends the testing of our codes beyond the independently performed calcula- 

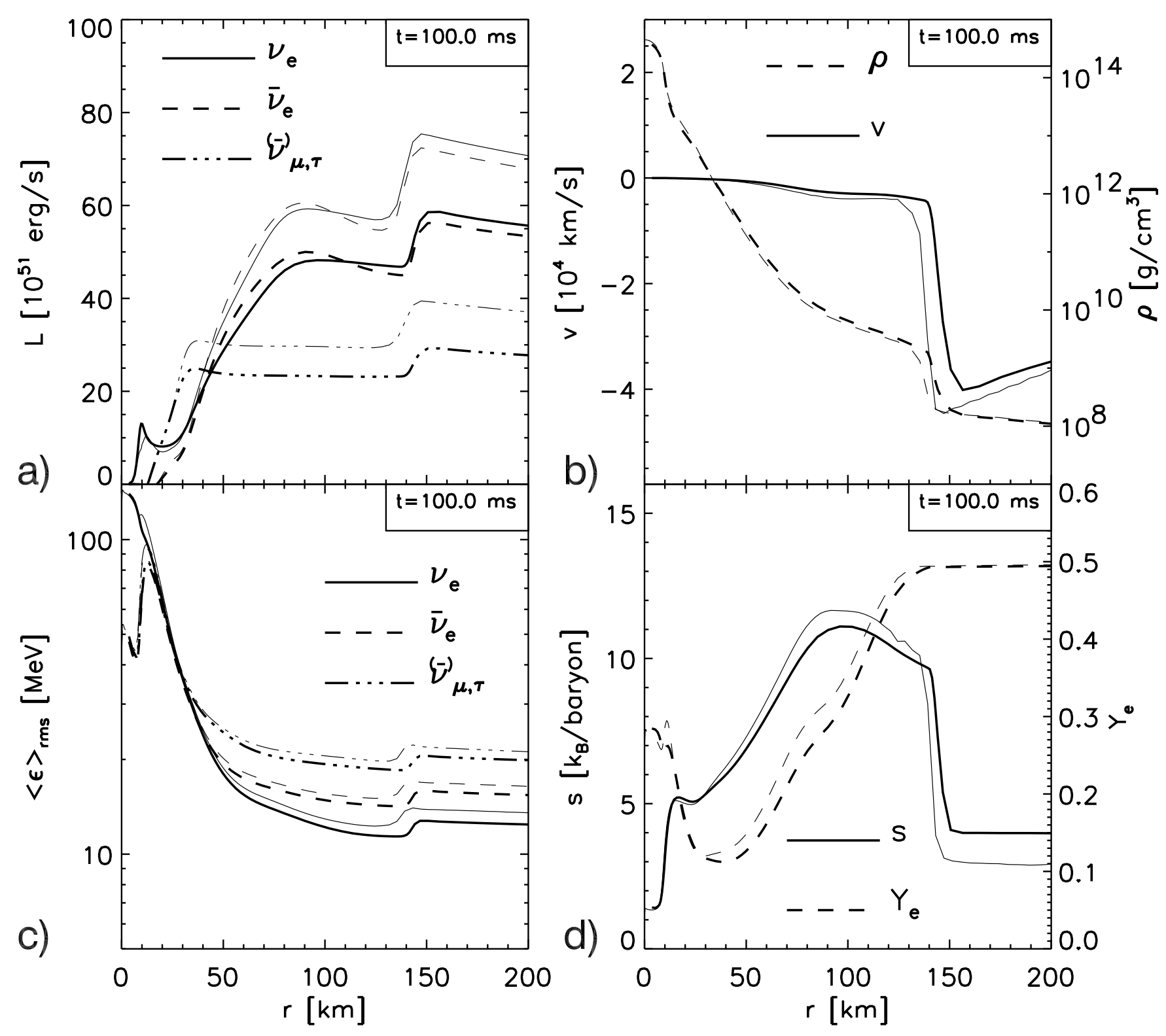

FIG. 11.- Snapshots at $100 \mathrm{~ms}$ after bounce for model G15. Data from the AGILE-BOLTZTRAN simulation are drawn with thick lines. Data from the verTex simulation are drawn with thin lines. Panel (b) shows the velocity (solid lines) and density (dashed lines) profiles, panel (d) the entropy (solid lines) and electron fraction (dashed line). The neutrino luminosities and rms energies are displayed in panels (a) and (c), respectively. Solid lines correspond to electron neutrinos, dashed lines to electron antineutrinos, and dash-dotted lines to $\mu$ or $\tau$-neutrinos (or their antiparticles). In this time slice we find a smaller shock position, somewhat faster infall ahead of the shock, and higher postshock infall velocities in VERTEX compared to the results of AGILE-BOLTZTRAN. Consistent with the more compact structure of the protoneutron star, the entropies, neutrino luminosities, and neutrino rms energies in VERTEX are larger than in AGILE-BOLTZTRAN.

tions of idealized problems which have analytical solutions (Messer 2000; Rampp \& Janka 2002; Liebendörfer, Rosswog. \& Thielemann 2002; Liebendörfer et al. 2004). Here we directly compare the results of the codes in the application they were actually developed for. Our aim was to assess quantitative differences in complex supernova simulations to reduce the probability of qualitative differences in future appli- cations. We also intended to create points of reference for future testing of codes that handle the challenges of supernova physics, and to lay the foundations for performing such tests in a more realistic way than by means of comparison with analytic solutions of idealized test problems.

We have encountered two fundamental difficulties in our comparison. The first is the fact that the two codes 

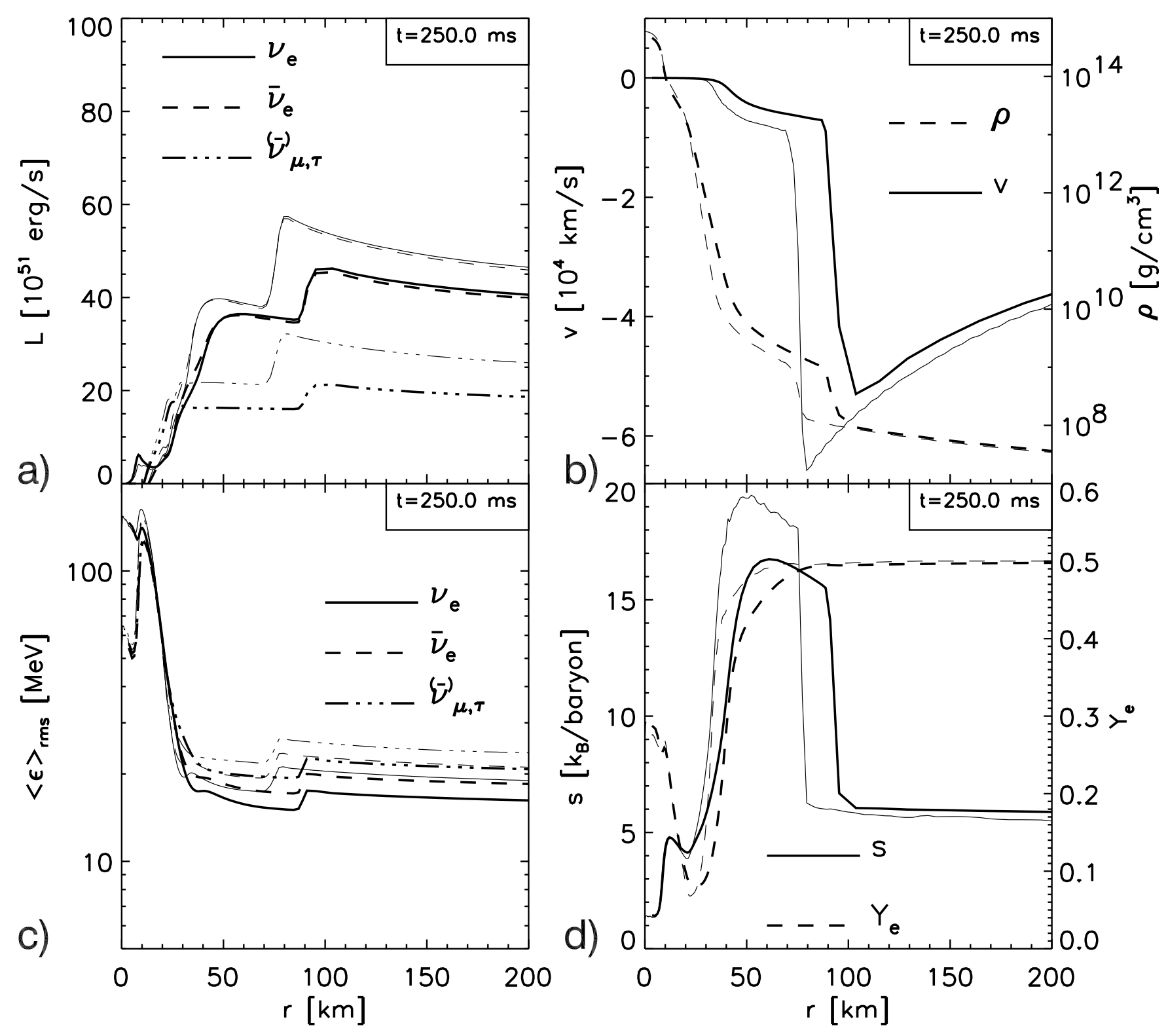

FIG. 12.- Snapshots at $250 \mathrm{~ms}$ after bounce for model G15. Data from the AGILE-BOLTZTRAN simulation are drawn with thick lines. Data from the verTex simulation are drawn with thin lines. Panel (b) shows the velocity (solid lines) and density (dashed lines) profiles, panel (d) the entropy and electron abundances. The neutrino luminosities and rms energies are displayed in panels (a) and (c). Solid lines correspond to electron neutrinos, dashed lines to electron antineutrinos, and dash-dotted lines to $\mu$ - or $\tau$-neutrinos (or their antiparticles). The protoneutron star in the VERTEX simulation is more compact than in the AGILE-BOLTZTRAN simulation.

employ different methods, use different basic quantities, and are differently structured. The comparison of the results of the two approaches is straightforward, but it is by far more challenging (and sometimes impossible) to track differences back to their origin if the compared quantities are not calculated in a similar way. The second difficulty is more related to supernova physics than to the methodology. In the comparison of our results we have very often encountered the situation that all quanti- ties within either one simulation are perfectly consistent, but still not the same as in the other simulation. Further investigations of the differences revealed small initial differences in several tightly coupled quantities that grow with ongoing evolution. Because of the strong feedbacks in the supernova problem it was often almost impossible to separate cause and consequences of the deviations. This strong coupling between quantities indicates that the problem is governed by many equilibria. Sometimes 


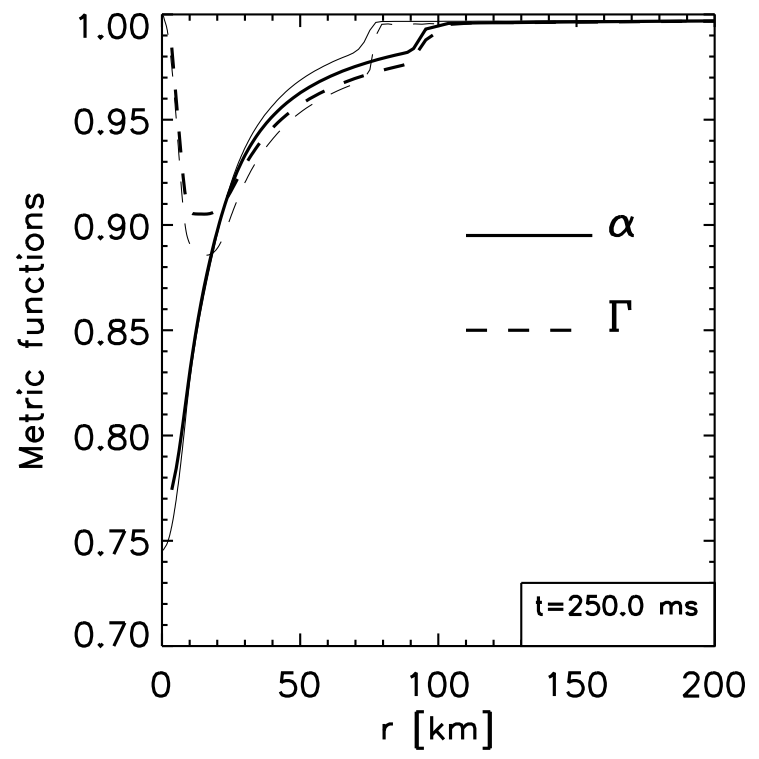

FIG. 13.- Metric coefficients $\alpha=g_{t t}^{1 / 2}$ and $\Gamma=g_{a a}^{-1 / 2} \partial r / \partial a=$ $\left[1+(u / c)^{2}-2 G m /\left(r c^{2}\right)\right]^{1 / 2}$ in the G15 run at $250 \mathrm{~ms}$ after bounce. The discontinuity of the lapse function across the shock front reflects the fact that the comoving frame is not an inertial system in the special relativistic limit. Results from AGILE-BOLTZTRAN are plotted with tick lines, those from VERTEX with thin lines. The higher compactness of the neutron star in the VERTEX run is obvious. Ahead of the shock metric effects are very small.

the results converge again and find back to a similar evolution as soon as the equilibrium is achieved. This is for example the case in model N13 when stationary-state accretion is established after the very dynamical early postbounce phase.

When we consider just the overall dynamical evolution of the spherically symmetric postbounce phases in Figs. [5] and 10] we find agreement in all qualitative features of the history. But we also find some significant quantitative differences. For example, the early shock propagation and the luminosities during the first $100 \mathrm{~ms}$ after bounce are different in model N13. We attribute an important part of these differences to the hydrodynamical shock propagation that appears to maintain a stronger shock in VERTEX than in AGILE-BOLTZTRAN. The close coincidence of the neutrino burst with the transition from a dynamical to an accretion shock in this model leads to an amplification of existing differences. The region behind the weaker shock in AGILE-BOLTZTRAN deleptonizes to a larger extent than behind the slightly stronger shock in VERTEX. While the deleptonization burst is more extended in AGILE-BOLTZTRAN, the VERTEX core could maintain higher luminosities later on. This may be the reason for a somewhat more optimistic shock propagation in the early phase of the VERTEX simulation of model N13.

No such amplification effect takes place in the G15 model where the shocks in both runs have already made the transition to an accretion front when they reach densities from where neutrinos begin to escape. Despite of some differences in the time-dependence of the shock strength, the evolution of these two more realistic runs with "standard" input physics agrees nicely during the early post-bounce phase. We find good agreement of the neutrino quantities in the diffusive inner core of the protoneutron star and the timing and peak height of the neutrino burst. The approximations of general relativistic effects in VERTEX yield accurate results until bounce and do not introduce larger uncertainties with respect to the general relativistic approach than other acceptable approximations. Differences appear only in the later evolution when the outer layers of the progenitor fall into the stalled accretion front. Some of these differences are caused by a different description of nuclear burning in both codes and a different capability to track the composition interfaces in the outer layers of the collapsing core. The main reason for the differences, however, is the approximate treatment of general relativity in the VERTEX simulation. The basically Newtonian hydrodynamics code employs a relativistically modified gravitational potential which in principle allows one to accurately describe hydrostatic configurations according to the TOV equation (Rampp \& Janka 2002). But the code disregards the effects of relativistic kinematics which causes an overestimation of the infall velocity ahead of the shock. The corresponding differences in the dynamical evolution feed back into the gravitational potential in a nonlinear way. This leads to a slightly more compact neutron star, a somewhat smaller radius of the accretion front, and a faster infall of matter between shock and neutron star, producing up to $\sim 20 \%$ higher accretion luminosities and rms energies of neutrinos and antineutrinos of all flavors. While most of these discrepancies in the neutrino quantities are a consequence of the different structure of the accretion layer in the VERTEX and AGILE-BOLTZTRAN simulations, a smaller contribution may also be ascribed to the fact that VERTEX takes into account general relativistic redshift, but ignores the metric effects in the radial coordinate.

The overall evolution, however, is consistent between both runs also in case of the relativistic G15 model. Even more, both computations produce not only a remarkable qualitative similarity of the behavior during all phases but also show nice agreement in most features of the radial profiles of the important quantities. This result may be especially useful for multi-dimensional simulations, where essential general relativistic effects should not be ignored, but a full relativistic treatment might not have highest priority.

Both methods have their vulnerabilities and some of them have led to lively discussions in the past. AGILEBOLTZTRAN was criticized because of the rigorous approach and the generous consumption of computer memory and CPU time. The resolution of the neutrino phase space was considered to be at the lower justifiable limit in earlier simulations. And indeed, the certainty that the evolution follows basic physical principles independently of the resolution must be earned by very specific twists and wrinkles in the finite difference representation (Liebendörfer et al. 2004). VERTEX has raised concerns with regard to consistency as it uses disjunct gridding for radiation transport and hydrodynamics and applies regridding procedures after bounce. The separate solution of the transport equations for neutrino number and neutrino energy only adds to the complexity. However, we 
did not find discrepancies in our comparison that would support any of these concerns to a degree that would question the reliability of the qualitative results of our explosion-free supernova simulations.

The different strengths of the codes are more visible in the quantitative details of the calculations. VERTEX produces great angular resolution in the flux factors even far from the neutrinospheres and keeps properly track of the sharp discontinuities in the composition of the outer layers. Its extendibility to two-dimensional simulations is built-in (Rampp \& Janka 2002) and the general relativistic approximation can be expected to produce good results in multi-dimensional situations as well (Buras et al. 2003). However, the solution of a model Boltzmann equation is much more involved in more than one dimension. Additional approximations introduced by spherical averaging or ray-by-ray techniques cannot be tested in a comparison between spherically symmetric models. If these approximations are good, the variable Eddington factor approach is a very efficient technique for multidimensional simulations.

AGILE-BOLTZTRAN demonstrates that the solution of only one transport equation for the neutrino distribution function can provide accurate radiation transport solutions in the diffusion limit and semi-transparent regime. Number and energy conservation are reasonably well fulfilled and the description of hydrodynamics and radiation transport add up to one consistent general relativistic finite difference representation of radiation hydrodynamics in spherical symmetry (Liebendörfer et al. 2004). The approach is in principle extendible to two and three dimensions and allows for adaptive zoning because it is based on the local description of the transport equation in confined fluid elements. But consistency is easily lost in higher dimensions (Cardall \& Mezzacappa 2003) and computer performance may become prohibitive in an implicit multidimensional discrete ordinates approach.

\section{CONCLUSIONS}

We have compared two different approaches to implement Boltzmann neutrino transport in spherically symmetric radiation hydrodynamics simulations of stellar core collapse and postbounce evolution. We performed calculations for two different progenitor stars, the 13 $\mathrm{M}_{\odot}$ progenitor of Nomoto \& Hashimoto (1988) and the $15 \mathrm{M}_{\odot}$ progenitor of Wooslev \& Weaver (1995). We present one Newtonian calculation (N13) with the minimum input physics that leads to a plausible scenario after bounce and a second relativistic calculation (G15) with the "standard" physics used in many recent supernova simulations. We find similar agreement in both cases. The reduced complexity of the input physics in the N13 model helps to isolate differences in the implementation of the hydrodynamics and the neutrino transport. We could improve the agreement by upgrading the first order donor-cell advection scheme in the implicit hydrodynamics code AGILE to a second order TVD advection scheme. The version with first order advection led to a more pessimistic shock propagation during the first $10 \mathrm{~ms}$ after bounce. It did, however, reveal an interesting relationship between the transition of the propagating discontinuity from a dynamical shock to an accretion front and the almost coincident launch of the neutrino burst.

A neutrino burst radiated from an accretion front maintains a high luminosity for a longer time than a neutrino burst produced by a dynamical shock, because an accretion front compresses matter at steady-state like conditions whereas the layer behind a dynamical shock gets diluted quickly so that electron captures diminish on a short timescale. Therefore less lepton number is lost in neutrinos from a dynamical shock which rapidly crosses the neutrinospheres, but neutrinos extract more leptons from the compressed matter behind the accretion front once the shock has stalled (i.e., the postshock velocity has become negative). This effect, however, turned out to produce transient differences only for a few milliseconds in our simulations, and convergence of the shock trajectories was found again after the shocks in both runs had transformed to accretion fronts. While the optimistic N13 model with only one neutrino flavor $\left(\nu_{e}\right.$ and $\left.\bar{\nu}_{e}\right)$ represents a case where the neutrinospheres are crossed by a dynamical shock, the relativistic model G15 serves as an example where the shock forms at a smaller enclosed mass due to the deeper general relativistic potential and where additional losses occur by the emission of $\mu$ - and $\tau$-neutrinos from deeper layers. In this case the shock turns into an accretion front before or at the time the neutrino burst is launched.

The overall evolution of both models is in good agreement when simulations with the two codes are compared. Differences in details were found, e.g., a slightly different shock propagation in the early hydrodynamical phase and more smearing of the composition interfaces in the outer progenitor layers by artificial diffusion in the case of AGILE. The luminosities in VERTEX tend to be slightly higher than in AGILE-BOLTZTRAN and the rms energies a little lower in the N13 model. The approximation of general relativistic effects by a modified gravitational potential in otherwise Newtonian hydrodynamics in VERTEX is very accurate up to bounce. In comparison with the general relativistic simulation of AGILE-BOLTZTRAN, however, a somewhat deeper potential associated with higher accretion rates develops during the long-term postbounce evolution. The consequence are larger neutrino luminosities and rms energies. But in general, good qualitative and satisfactory quantitative agreement of all important temporal and radial features was found also in the relativistic model. Major differences can result from implementation-specific rather than from methodspecific details, e.g. from the former use of a low-order advection scheme in AGILE-BOLTZTRAN or from the specific choice of the finite differencing in both codes.

We come to the conclusion that both methods work satisfactorily well in this application and give comparable results. We determined similar computational needs for our not thoroughly optimized codes. Standard runs with AGILE-BOLTZTRAN tend to consume slightly less computer time. But standard runs with VERTEX have been performed with better energy resolution and the angular resolution that can be achieved at larger radii is out of reach for $S_{N}$ methods. Hence, a detailed comparison of CPU time requirements is not really meaningful. Moreover, faster methods may have been developed in the meantime (Burrows et al. 2000; Thompson, Burrows, \& Pinto 2003). Rather than arguing about the "best" method for a certain application, we recommend to pursue a variety of feasible numerical approaches for future astrophysical simulations, opening 
up the possibility of independent mutual validation of the results. We hope that our comparison provides a useful step towards quantitative modeling of a very complex astrophysical problem.

\section{ACKNOWLEDGMENTS}

We thank R. Buras and O. E. B. Messer for many important contributions to VERTEX and AGILE-BOLTZTRAN respectively, in particular for implementing the threeflavor versions of the codes and coining subroutines to calculate the neutrino pair and bremsstrahlung rates. We are grateful for the most recent subroutine for ion-ion correlations provided to us by N. Itoh and collaborators. We are greatly indebted to Chris Fryer for suggesting and promoting this comparison. The Institute for Nuclear Theory at the University of Washington is acknowledged for its hospitality and the Department of Energy for support during a visit of the Summer Program on Neutron Stars in 2001, during which we started this work. AM and ML acknowledge funding by the NSF under contract AST-9877130, the Oak Ridge National Laboratory, managed by UT-Batelle, LLC, for the U.S. Department of Energy under contract DE-AC05-00OR22725, and the DoE HENP SciDAC Program. HTJ and MR are grateful for support by the Sonderforschungsbereich 375 on "Astroparticle Physics" of the Deutsche Forschungsgemeinschaft. The computations of the Garching group were mostly performed on the CRAY T90 and CRAY SV1ex of the John von Neumann Institute for Computing (NIC) in Jülich and the computations of the Oak Ridge-Basel group on the CITA Itanium I.

\section{REFERENCES}

Bethe, H. A. \& Wilson, J. R. 1985, ApJ, 295, 14

Bludman, S. A. \& Cernohorsky, J. 1995, Phys. Rep., 256, 37

Bruenn, S. W. 1985, ApJS, 58, 771

Bruenn, S. W. \& Mezzacappa, A. 1997, Phys. Rev. D, 56, 7529

Bruenn, S. W., DeNisco, K. R., \& Mezzacappa, A. 2001, ApJ, 560, 326

Buras, R., Rampp, M., Janka, H.-Th., \& Kifonidis, K. 2003, Phys. Rev. Lett., 90, 241101

Burrows, A., Young, T., Pinto, P., Eastman, R., \& Thompson, T. A. 2000, ApJ, 539, 865

Calder, A. C., Fryxell, B., Plewa, T., Rosner, R., Dursi, L. J., Weirs, V. G., Dupont, T., Robey, H. F., Kane, J. O., Remington, B. A., Drake, R. P., Dimonte, G., Zingale, M., Timmes, F. X., Olson, K., Ricker, P., MacNeice, P., Tufo, H. M. 2002, ApJS, 143, 201

Cardall,C. Y., Mezzacappa, A. 2003, Phys. Rev. D, 68, 023006

Cernohorsky, J. 1994, ApJ, 433, 247

Colella, P. \& Woodward, P. 1984, J. Comp. Phys., 54, 174

Dorfi, E. A., \& Drury, L. O'C. 1987, J. Comput. Phys., 69, 175

Einfeldt, B. 1988, SIAM Jour. Numer. Anal., 25, 294

Fryxell, B., Müller, E., \& Arnett, W. 1989, Hydrodynamics and Nuclear Burning, preprint MPA-449, Max Planck Institut für Astrophysik, Garching

Horowitz, C. 1997, Phys. Rev. D, 55, 4577

Itoh, N. 1975, Prog. Theor. Phys., 54, 1580

Itoh, N., Asahara, R., Tomizawa, N., Wanajo, S., Nozawa, S. 2004, astro-ph/0401488

Janka, H.-Th. 2001, A\&A, 368, 527

Janka, H.-Th., Buras, R., Kifonidis, K., Plewa, T., \& Rampp, M. 2004, in Stellar Collapse, ed. by Fryer, C. L. (Dordrecht, the Netherlands: Kluwer Academic Publishers), 65

Keil, W. 1997, PhD thesis, Technische Universität München

Kifonidis, K. 2000, PhD thesis, Technische Universität München

Lattimer, J. \& Swesty, F. D. 1991, Nucl. Phys., A535, 331

Liebendörfer, M. 2000, Ph.D. thesis (Basel: University of Basel)

Liebendörfer, M., Mezzacappa, A., \& Thielemann, F.-K. 2001, Phys. Rev., D63, 104003

Liebendörfer, M., Mezzacappa, A., Thielemann, F.-K., Messer, O. E. B., Hix, W. R., \& Bruenn, S. W. 2001, Phys. Rev., D63 103004

Liebendörfer, M., Rosswog, S. K., \& Thielemann, F.-K. 2002, ApJS, 141, 229

Liebendörfer, M., Messer, O. E. B., Mezzacappa, A., Bruenn, S. W. Cardall, C. Y., \& Thielemann, F.-K. 2004, ApJS, 150, 263
Lindquist, R. W. 1966, Ann. Phys., 37, 487

May, M. M., \& White, R. H. 1966, Phys. Rev., 141, 1232

Messer, O. E. B. 2000, Ph.D. thesis (Knoxville: University of Tennessee)

Mezzacappa, A. \& Bruenn, S. W. 1993, ApJ 405, 669

Mezzacappa, A. \& Bruenn, S. W. 1993, ApJ 405, 637

Mezzacappa, A. \& Bruenn, S. W. 1993, ApJ 410, 740

Mezzacappa, A., Liebendörfer, M., Messer, O. E. B., Hix, W. R., Thielemann, F.-K., \& Bruenn, S. W. 2001, PRL, 86, 1935

Mezzacappa, A. \& Messer, O. E. B. 1999, JCAM, 109, 281

Misner, C. W., \& Sharp, D. H. 1964, Phys. Rev., B136, 571

Myra, E. S., Bludman, S. A., Hoffman, Y., Lichtenstadt, I., Sack, N., \& Van Riper, K. A. 1987, ApJ, 318, 744

Nomoto, K. \& Hashimoto, M. 1988, Phys. Rep., 163, 13

Plewa, T. \& Müller, E. 1999, A\&A, 342, 179

Plewa, T. \& Müller, E. 2001, Computer Physics Communications, 138,101

Quirk, J. J. 1994, Int. J. Num. Meth. Fluids, 18, 555

Rampp, M. 2000, PhD thesis, Technische Universität München

Rampp, M. \& Janka, H. T. 2000, ApJL, 539, L33

Rampp, M. \& Janka, H. T. 2002, A\&A, 396, 361

Smit, J. M., Cernohorsky, J., \& Dullemond, C. P. 1997, A\&A, 325, 203

Sumiyoshi, K., Suzuki, H., Otsuki, K., Terasawa, M., \& Yamada, S. 2000, Publ. Astron. Soc. Jap., 52, 601

Takahashi, K., Witti, J., \& Janka, H.-T. 1994, A\&A, 286, 857

Thompson, T. A., Burrows, A., \& Meyer, B. S. 2001, ApJ, 562, 887

Thompson, T. A., Burrows, A., \& Pinto, P. A. 2003, ApJ, 592, 434

Tscharnuter, W. M., \& Winkler, K. H. 1979, Comput. Phys. Comm., 18, 171

Wanajo, S., Kajino, T., Mathews, G. J., \& Otsuki, K. 2001, ApJ, 554,578

Wilson, J. R. 1985, in Numerical Astrophysics, ed. by Centrella J. M., LeBlanc, J. M., \& Bowers, R. L. (Boston: Jones and Bartlett), 422

Winkler, K.-H., Norman, M. L., \& Mihalas, D. 1984, J. Quant. Spectrosc. Radiat. Transf., 31, 473

Woosley, S. E., Wilson, J. R., Mathews, G. J., Hoffman, R. D., \& Meyer, B. S. 1994, ApJ, 433, 229

Woosley, S. E., Weaver, T. A. 1995, ApJS, 101, 181 